\title{
Unexpected Synthesis, Single-Crystal X-ray Structure, Anticancer Activity, and Molecular Docking Studies of Certain 2-((Imidazole/Benzimidazol-2-yl)thio)- 1-arylethanones
}

\author{
Tarfah Al-Warhi ${ }^{1}$, Mohamed A. Said ${ }^{2}$, Mahmoud A. El Hassab ${ }^{3}{ }^{(\mathbb{C},}$ Nada Aljaeed ${ }^{1}$, \\ Hazem A. Ghabour ${ }^{4}$ (D), Hadia Almahli ${ }^{5,6}$, Wagdy M. Eldehna ${ }^{7, *(D)}$ and \\ Hatem A. Abdel-Aziz ${ }^{8, *(D)}$ \\ 1 Department of Chemistry, College of Science, Princess Nourah bint Abdulrahman University, \\ Riyadh 11671, Saudi Arabia; tarfah-w@hotmail.com (T.A.-W.); noaljaeed@pnu.edu.sa (N.A.) \\ 2 Department of Pharmaceutical Chemistry, Faculty of Pharmacy, Egyptian Russian University, \\ Badr City 11829, Cairo, Egypt; madyyy_@hotmail.com \\ 3 Department of Pharmaceutical Chemistry, School of Pharmacy, Badr University in Cairo, \\ Badr City 11829, Cairo, Egypt; elhasabma85@gmail.com \\ 4 Department of Medicinal Chemistry, Faculty of Pharmacy, Mansoura University, Mansoura 35516, Egypt; \\ ghabbourh@yahoo.com \\ 5 Department of Chemistry, Chemistry Research Laboratory, University of Oxford, 12 Mansfield Road, \\ Oxford OX1 3TA, UK; hadia.almahli@yahoo.com \\ 6 Department of Chemistry, School of Life Sciences, University of Sussex, Sussex \\ House, Falmer, Brighton BN1 9RH, UK \\ 7 Department of Pharmaceutical Chemistry, Faculty of Pharmacy, Kafrelsheikh University, \\ Kafrelsheikh 33516, Egypt \\ 8 Department of Applied Organic Chemistry, National Research Center, Dokki, Giza, P.O. Box 12622, Egypt \\ * Correspondence: wagdy2000@gmail.com (W.M.E.); hatem_741@yahoo.com (H.A.A.-A.)
}

Received: 29 April 2020; Accepted: 25 May 2020; Published: 31 May 2020

\begin{abstract}
In connection with our research program concerning development of novel effective benzimidazole-based anticancer candidates, herein we describe a new unexpected synthetic route to obtain a series of 2-((imidazole/benzimidazol2-yl)thio)1-arylethanones endowed with promising anti-breast cancer and Cyclin-dependent kinase 2 (CDK2) inhibitory activities. Contrary to expectations, products for the reaction of 2 -mercaptoimidazole/benzimidazole $2 \mathrm{a}, \mathrm{b}$ with $\beta$-keto esters $6 a-c$ were unambiguously assigned as $2-(($ imidazol/benzimidazol2-yl)thio) 1 -arylethanones 10a-f based on NMR spectroscopy and single-crystal X-ray crystallographic analyses. In vitro anticancer activities for herein reported imidazole/benzimidazoles 10a-f were assessed through a cell-based assay against human breast cancer T4-7D and MCF-7 cell lines. Benzimidazoles 10d-f exerted better anti-proliferative action towards T4-7D and MCF-7 cell lines than their corresponding imidazole counterparts 10a-c. Furthermore, a molecular docking study suggested CDK2 kinase as a potential enzymatic target for benzimidazoles $10 \mathrm{~d}-\mathrm{f}$, and investigated their possible binding pattern and interactions within CDK2 active site. Thereafter, benzimidazoles $10 \mathrm{~d}-\mathrm{f}$ were in vitro examined for their CDK2 inhibitory action, where they exerted good activity. Finally, several key ADME and druglikeness properties were predicted by the SwissADME online tool. Interestingly, benzimidazoles $10 \mathrm{~d}-\mathrm{f}$ were found to have no violations in all druglikeness rules (Veber, Lipinski, Ghose, Muegge, and Egan). In addition, they had neither PAINS nor structural alerts (Brenks). In conclusion, benzimidazoles $10 \mathrm{~d}-\mathrm{f}$ demonstrated not only a promising anticancer activities but also an acceptable ADME and physicochemical properties especially benzimidazole 10e.
\end{abstract}


Keywords: imidazole/benzimidazole; $\beta$-keto esters; anticancer; cdk2 inhibitors; single-crystal X-ray analyses

\section{Introduction}

On account of their existence in several natural bioactive compounds and some naturally occurring nucleotides in addition to their significant roles as therapeutics in diverse clinical applications, imidazole and benzimidazole are occupying a featured position in the synthetic medicinal chemistry [1-4].

Imidazoles and benzimidazoles are quite unique and universally privileged scaffolds, which are core units in numerous drugs and drug candidates [5] such as angiotensin II receptor antagonists, $\mathrm{H}^{+} / \mathrm{K}^{+}$-ATPases inhibitors, $\mathrm{H}_{1}$-antihistamines, anthelmintics, and anticancer agents. Accordingly, medicinal chemists embarked on developing and exploring varied imidazole- and benzimidazole-based small molecules to afford novel and promising biologically active compounds. In this context, imidazoles and benzimidazoles emerged as important structural motifs for anticancer drug discovery and development with diverse potential cellular and enzymatic targets [6-10].

In recent years, there are several innovative, efficient, and mild synthetic protocols and routes that have been reported for the development of benzimidazole-based small molecules. Some studies reported the synthesis of benzimidazoles by the use of water as the reaction medium [11,12]. In addition, many other green synthetic methods have been reported [13-15]. Moreover, a large number of studies utilized different catalysts to synthesize diverse benzimidazole derivatives [16], to name just a few, the palladium-catalyzed [17], zinc-catalyzed [18], erbium-catalyzed [14], and copper-catalyzed synthesis [19].

In the last few years, we have reported two studies to investigate the antitumor actions for a series of 232 -((benzimidazol2-yl)thio)1-arylethanone derivatives (structures 1, Figure 1) through different cell-based assays $[20,21]$. The first study revealed the capability of these benzimidazole derivatives to affect the bulk of tumor cells and cancer stem cells via inhibition of growth of the HT29 colon cancer cells and the CD133 cell-surface expression, a cancer stem cell marker, in HT2-9 cells [20]. In the second study, the explored benzimidazole derivatives 1 (Figure 1) displayed promising anticancer action toward triple-negative MDAMB4-68 breast cancer cell line, in addition to induction of apoptosis in the same cells. In both previous studies, the target benzimidazole derivatives 1 were prepared through reaction of 2-mercaptobenzimidazole $2 \mathrm{~b}, \mathrm{R}=-(\mathrm{CH})_{4}{ }^{-}$, with different acetophenones in boiling glacial acetic acid and two equivalents of concentrated sulfuric acid, with sequent neutralization for the produced sulfate salts yielding 2-((benzimidazol2-yl)thio)1-arylethanones 3 in a good yield [20,21] (Figure 1).

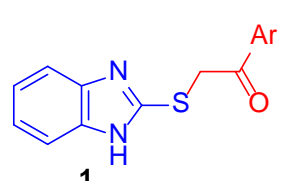

1

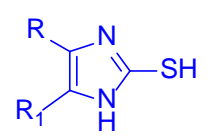

2a, $\mathrm{R}=\mathrm{H}$ $\mathbf{2 b}, \mathrm{R}=-(\mathrm{CH}) 4$<smiles>Brc1csc2nc3ccccc3n12</smiles>

3

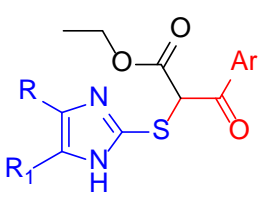

4a-f

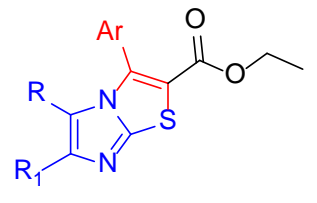

5a-f

Figure 1. Chemical structure of compounds 1-5.

In 2017, we reported another study that adopted a structural rigidification approach to develop new set of 3-arylthiazolo[3,2-a] benzimidazole derivatives (structure 3, Figure 1) as rigid analogues to the previously reported 2-((benzimidazol2-yl)thio)1-arylethanones 1 [22]. The prepared arylthiazolo[3,2-a]benzimidazole derivatives exerted efficient growth-inhibitory action against triple-negative MDAMB4-68 breast cancer and HT29 colon cancer cell lines in addition to their capability to inhibit the CD133 cell surface expression. This arylthiazolo[3,2-a]benzimidazole series 
was prepared through a one-pot, two-components heterocyclization reaction, via the reaction of 2 -mercaptobenzimidazole $2 \mathrm{~b}$ with different acetophenones in boiling glacial acetic acid and five equivalents from concentrated sulfuric acid [22].

Based on the reported effective antitumor activities for both 2-((benzimidazol2-yl)thio)1arylethanones (1) as well as 3-arylthiazolo[3,2-a]benzimidazole derivatives (3) series [20-22], in the current research work we aspired to develop a novel series of imidazole/benzimidazole-based esters 4 and their cyclized analogues 5 (Figure 1) via the reaction of imidazole/benzimidazole thiols $2 \mathrm{a}, \mathrm{b}$ with different $\beta$-keto esters in boiling glacial acetic acid, in the presence of two and five equivalents of concentrated sulfuric acid, respectively, following the above-mentioned procedure [20-22]. Interestingly, this reaction afforded an unexpected sulfate salts 9 (Scheme 1) rather than sulfate salts for 4 or 5 . The latter sulfate salts 9 were then neutralized to produce 2- ((benzimidazol2-yl)thio)1-arylethanones 10 (Scheme 1). This reaction, as well as its proposed pathway, will be discussed in this study in the light of X-ray single-crystal analysis for $9 \mathrm{~b}$ and $10 \mathrm{f}$.

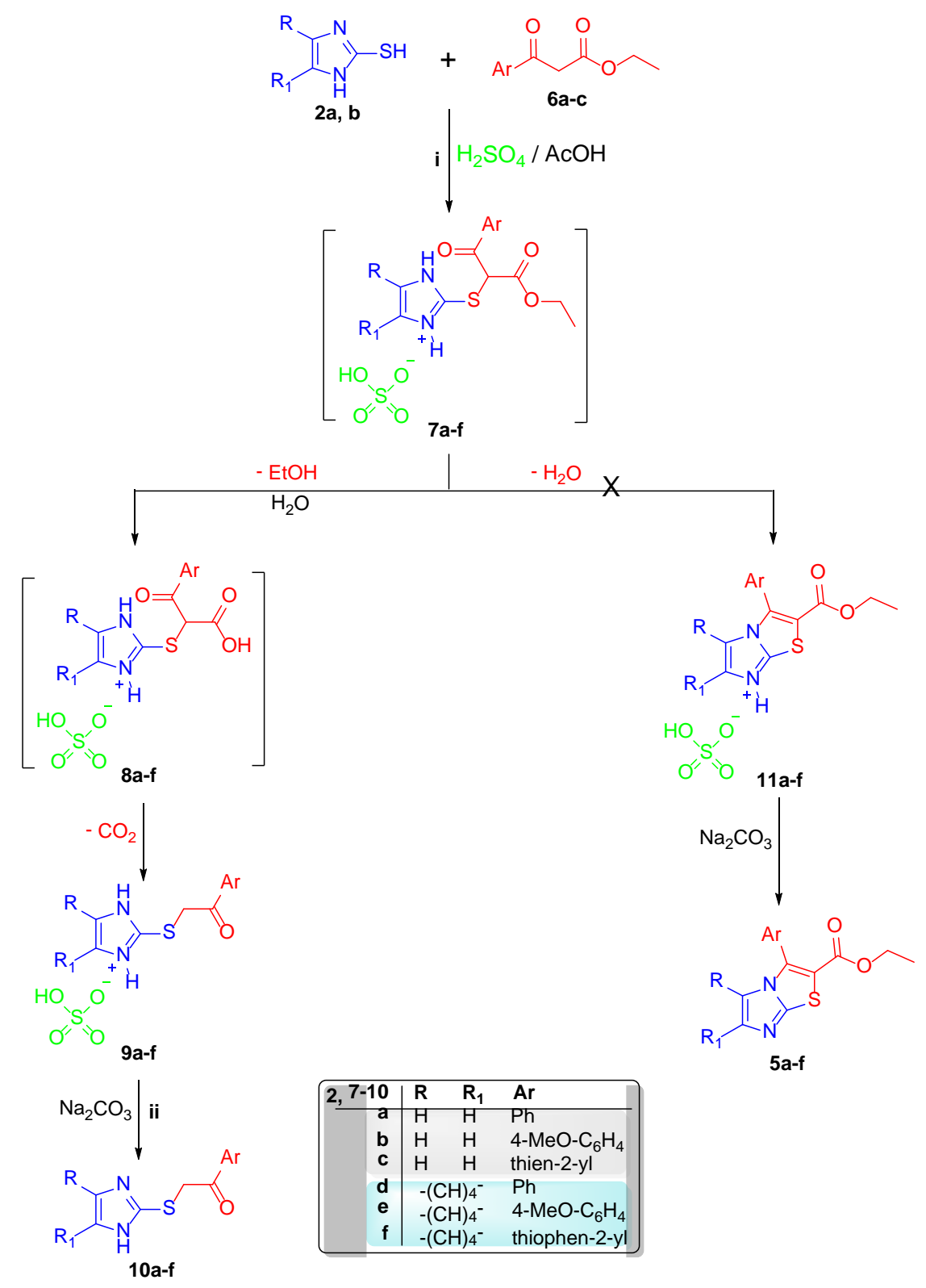

Scheme 1. Reaction of imidazole/benzimidazole thiols $\mathbf{2 a}$, b with $\beta$-keto esters $\mathbf{6 a}-\mathbf{c}$ (i) reflux for $1 \mathrm{~h}$, (ii) stirring at room temperature for $3 \mathrm{~h}$. 
Moreover, the anticancer activities for herein-reported imidazole/benzimidazole derivatives 10a- $\mathrm{f}$ will be assessed through a cell-based assay (against human breast cancer T4-7D and MCF-7 cell lines) as well as through a cell-free assay (against Cyclin-dependent kinase 2, CDK2) to explore the potential enzymatic targets for the prepared derivatives 10a-f. Also, several key physicochemical parameters will be calculated in order to explore their pharmacokinetics and druglikeness properties.

\section{Results and Discussion}

\subsection{Chemistry}

This study describes the reaction of 2-mercaptoimidazole (2a) and 2-mercaptobenzimidazole (2b) with $\beta$-keto esters $6 \mathrm{a}-\mathrm{c}$ in an attempt to obtain a new series of ethyl 2-((imidazol/benzimidazol2yl)thio)3-oxo3-arylpropanoates 4a-f or ethyl 3-aryl imidazol/benzimidazo[2,1- $b$ ] thiazole2-carboxylates $5 a-f$ (Scheme 1). The expected synthetic pathway for this reaction is depicted in Scheme 1. The conditions adopted for this reaction were heating under reflux in acetic acid and in the existence of two equivalents from $\mathrm{H}_{2} \mathrm{SO}_{4}(98 \%)$ followed by neutralization with sodium bicarbonate, which is similar to the reported conditions in our previous studies for the reaction of 2-mercaptobenzimidazole with different acetophenones [20,21].

As shown in Scheme 1, contrary to expectations, the products were unambiguously assigned as 2-((imidazol/benzimidazol2-yl)thio)1-arylethanones 10a-f instead of ethyl 2-((imidazol/benzimidazol2yl)thio)3-oxo3-arylpropanoates 4a-f or ethyl 3-aryl imidazol/ benzimidazo[2,1-b]thiazole2-carboxylates $5 \mathrm{a}-\mathrm{f}$, based on NMR spectroscopy and X-ray crystallographic analysis of sulfate salt $9 \mathrm{~b}$ and free base $10 \mathrm{f}$ (Scheme 1). The analytical data and physical characters of products $9 \mathrm{~d}-\mathrm{f}$ and $10 \mathrm{~d}-\mathrm{f}$ were similar to the products afforded from the reaction of the corresponding acetophenones with 2-mercapto-imidazole/benzimidazole 2a,b under the same reaction conditions (Scheme 1).

The proposed reaction pathway for this unexpected manner was suggested, as in Scheme 1, that an electrophilic attack between 2-mercaptoimidazole (2a) or 2-mercaptobenzimidazole (2b) and the alpha carbon of $\beta$-keto esters 6a-c took place, forming 2-((1-ethoxy1-, 3-dioxo3-arylpropan2-yl)thio)1- $H$-imidazol/benzimidazol3-ium hydrogen sulfate salts $7 a-f$. These salt intermediates were subjected to ester hydrolysis, affording the corresponding acid intermediates $8 \mathrm{a}-\mathrm{f}$, respectively, that subsequently decarboxylated to produce the salt products $9 \mathrm{a}-\mathrm{f}$. Neutralization of sulfate salts $9 \mathrm{a}-\mathrm{f}$ was performed via stirring with an aqueous solution of sodium hydrogen carbonate in order to yield 10a-f, respectively (Scheme 1).

The ${ }^{1} \mathrm{H}$ NMR spectra for imidazol/benzimidazoles 10a-f confirmed disappearance of the triplet and quartet signals attributable to the aliphatic protons in the ethyl ester functionality $\left(-\mathrm{COOC} \underline{\mathrm{H}}_{2} \mathrm{C}_{3}\right)$. In addition, they displayed the alpha-protons as singlet signals integrated for two protons of $-\mathrm{S}-\mathrm{CH}_{2}-$. Furthermore, ${ }^{13} \mathrm{C}$ NMR spectra for imidazole/benzimidazole-based derivatives 10a-f uncovered the disappearance of the carbons of the ethyl ester group $\left(-\underline{\mathrm{COOC}} \mathrm{H}_{2} \underline{\mathrm{C}} \mathrm{H}_{3}\right)$. Moreover, the single-crystal $\mathrm{X}$-ray analysis was utilized to confirm the structure of compounds $9 \mathrm{~b}$ and $10 \mathrm{f}$ unambiguously.

\subsection{Single-Crystal $X$-ray Crystallographic Analysis}

Crystallographic data for imidazole sulfate-salt $9 \mathrm{~b}$ and benzimidazole derivative $10 \mathrm{f}$ were assigned CCDC numbers 1524590 and 1429947, respectively, in the Cambridge Crystallographic Data Centre. The asymmetrical unit of imidazole sulfate-salt $9 \mathrm{~b}$ contained one molecule cation and one hydrogen sulfate anion to form a stable salt, Figure 2. Regarding benzimidazole derivative 10f, the unit cell contained only one independent base molecule. The bond lengths, as well as angles, were in the normal ranges [23]. Molecules of imidazole sulfate-salt $9 \mathrm{~b}$ were linked via five intermolecular hydrogen bonds in order to build a network structure (Figure 3) in the crystal packing. On the other hand, benzimidazole derivative $10 \mathrm{f}$ was packed together through one strong hydrogen bond along the $\mathrm{b}$ axis (Figure 4). Certain chosen bond lengths and angles, as well as hydrogen bond geometry of compounds $9 \mathrm{~b}$ and 10f, are listed in Tables 1 and 2, respectively. 


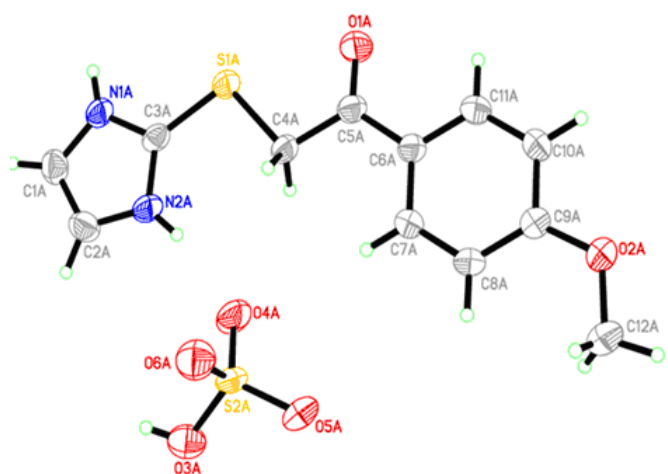

9b

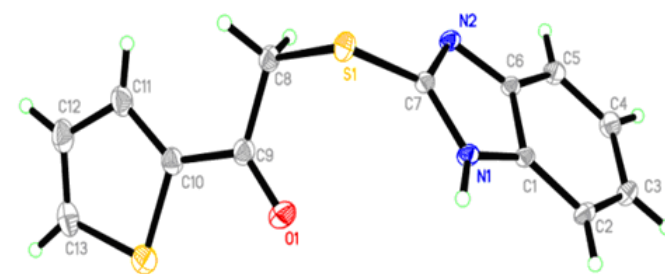

$10 f$

Figure 2. Oak Ridge Thermal Ellipsoid Plot (ORTEP) diagrams for imidazole sulfate-salt $\mathbf{9 b}$ and benzimidazole derivative 10f. Displacement ellipsoids have been plotted at the $40 \%$ probability level for non-H atoms.

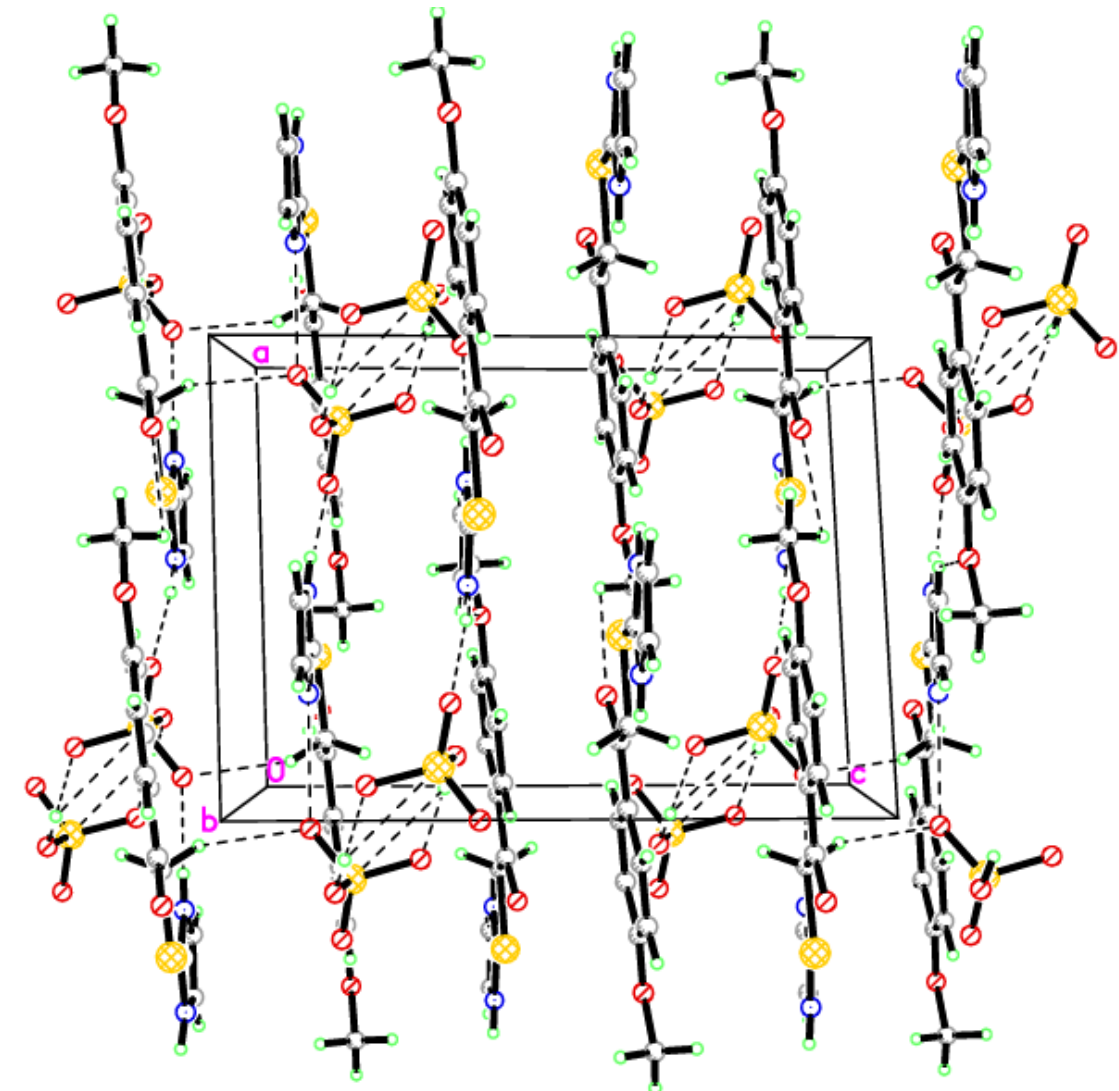

Figure 3. Molecular packing for imidazole sulfate-salt 9b, showing hydrogen bonds that were drawn as dashed lines forming a network structure.

\subsection{Biological Evaluation}

\section{Cytotoxic Action against Breast Cancer Cell Lines}

The potential anti-proliferative action toward two human breast cancer cell lines, T4-7D and MCF-7, was assessed for all the synthesized imidazole derivatives $(10 \mathrm{a}-\mathrm{c})$ and benzimidazole derivatives $(10 \mathrm{~d}-\mathrm{f})$ by the aid of Sulforhodamine B (SRB) assay [24] where the approved antitumor drug staurosporine was incorporated as a positive control in the experiments. The calculated $\mathrm{IC}_{50}$ values (a concentration demanded to inhibit proliferation of the tumor cells by $50 \%$ ) are displayed in Table 3. 
Table 1. Selected geometric parameters $\left(\AA,^{\circ}\right)$ of imidazole sulfate-salt $9 \mathbf{b}$ and benzimidazole derivative $\mathbf{1 0 f}$.

\begin{tabular}{|c|c|c|c|}
\hline \multicolumn{4}{|c|}{$9 b$} \\
\hline Bond & Å & Bond & $\AA$ \\
\hline $\mathrm{S} 1 \mathrm{~A}-\mathrm{C} 3 \mathrm{~A}$ & $1.732(2)$ & $\mathrm{O} 2 \mathrm{~A}-\mathrm{C} 12 \mathrm{~A}$ & $1.428(3)$ \\
\hline $\mathrm{S} 1 \mathrm{~A}-\mathrm{C} 4 \mathrm{~A}$ & $1.807(2)$ & $\mathrm{O} 2 \mathrm{~A}-\mathrm{C} 9 \mathrm{~A}$ & $1.354(3)$ \\
\hline $\mathrm{S} 1 \mathrm{~B}-\mathrm{C} 4 \mathrm{~B}$ & $1.805(2)$ & $\mathrm{O} 1 \mathrm{~B}-\mathrm{C} 5 \mathrm{~B}$ & $1.208(3)$ \\
\hline $\mathrm{S} 1 \mathrm{~B}-\mathrm{C} 3 \mathrm{~B}$ & $1.729(2)$ & $\mathrm{O} 2 \mathrm{~B}-\mathrm{C} 12 \mathrm{~B}$ & $1.433(3)$ \\
\hline $\mathrm{S} 2 \mathrm{~A}-\mathrm{O} 4 \mathrm{~A}$ & $1.4343(19)$ & $\mathrm{O} 2 \mathrm{~B}-\mathrm{C} 9 \mathrm{~B}$ & $1.357(3)$ \\
\hline $\mathrm{S} 2 \mathrm{~A}-\mathrm{O} 3 \mathrm{~A}$ & $1.5235(19)$ & $\mathrm{N} 1 \mathrm{~A}-\mathrm{C} 3 \mathrm{~A}$ & $1.324(3)$ \\
\hline $\mathrm{S} 2 \mathrm{~A}-\mathrm{O} 5 \mathrm{~A}$ & $1.4346(18)$ & $\mathrm{N} 1 \mathrm{~A}-\mathrm{C} 1 \mathrm{~A}$ & $1.376(3)$ \\
\hline S2A-O6A & $1.4764(18)$ & $\mathrm{N} 2 \mathrm{~A}-\mathrm{C} 2 \mathrm{~A}$ & $1.370(3)$ \\
\hline $\mathrm{S} 2 \mathrm{~B}-\mathrm{O} 4 \mathrm{~B}$ & $1.5283(17)$ & $\mathrm{N} 2 \mathrm{~A}-\mathrm{C} 3 \mathrm{~A}$ & $1.332(3)$ \\
\hline $\mathrm{S} 2 \mathrm{~B}-\mathrm{O} 5 \mathrm{~B}$ & $1.4782(18)$ & $\mathrm{N} 1 \mathrm{~B}-\mathrm{C} 3 \mathrm{~B}$ & $1.326(3)$ \\
\hline $\mathrm{S} 2 \mathrm{~B}-\mathrm{O} 6 \mathrm{~B}$ & $1.4330(18)$ & $\mathrm{N} 1 \mathrm{~B}-\mathrm{C} 1 \mathrm{~B}$ & $1.379(3)$ \\
\hline $\mathrm{S} 2 \mathrm{~B}-\mathrm{O} 3 \mathrm{~B}$ & $1.4346(17)$ & $\mathrm{N} 2 \mathrm{~B}-\mathrm{C} 2 \mathrm{~B}$ & $1.372(3)$ \\
\hline $\mathrm{O} 1 \mathrm{~A}-\mathrm{C} 5 \mathrm{~A}$ & $1.212(3)$ & $\mathrm{N} 2 \mathrm{~B}-\mathrm{C} 3 \mathrm{~B}$ & $1.329(3)$ \\
\hline Angle & $\circ$ & Angle & $\circ$ \\
\hline $\mathrm{C} 3 \mathrm{~A}-\mathrm{S} 1 \mathrm{~A}-\mathrm{C} 4 \mathrm{~A}$ & $99.79(10)$ & $\mathrm{N} 1 \mathrm{~A}-\mathrm{C} 1 \mathrm{~A}-\mathrm{C} 2 \mathrm{~A}$ & $106.5(2)$ \\
\hline $\mathrm{C} 3 \mathrm{~B}-\mathrm{S} 1 \mathrm{~B}-\mathrm{C} 4 \mathrm{~B}$ & $99.33(10)$ & $\mathrm{N} 2 \mathrm{~A}-\mathrm{C} 2 \mathrm{~A}-\mathrm{C} 1 \mathrm{~A}$ & $107.5(2)$ \\
\hline $\mathrm{O} 4 \mathrm{~A}-\mathrm{S} 2 \mathrm{~A}-\mathrm{O} 5 \mathrm{~A}$ & $112.49(11)$ & $\mathrm{N} 1 \mathrm{~A}-\mathrm{C} 3 \mathrm{~A}-\mathrm{N} 2 \mathrm{~A}$ & 107.11(19) \\
\hline $\mathrm{O} 4 \mathrm{~A}-\mathrm{S} 2 \mathrm{~A}-\mathrm{O} 6 \mathrm{~A}$ & $111.10(11)$ & $\mathrm{S} 1 \mathrm{~A}-\mathrm{C} 3 \mathrm{~A}-\mathrm{N} 1 \mathrm{~A}$ & $122.50(16)$ \\
\hline $\mathrm{O} 3 \mathrm{~A}-\mathrm{S} 2 \mathrm{~A}-\mathrm{O} 6 \mathrm{~A}$ & $106.18(10)$ & $\mathrm{S} 1 \mathrm{~A}-\mathrm{C} 3 \mathrm{~A}-\mathrm{N} 2 \mathrm{~A}$ & $130.35(16)$ \\
\hline $\mathrm{O} 5 \mathrm{~A}-\mathrm{S} 2 \mathrm{~A}-\mathrm{O} 6 \mathrm{~A}$ & $111.22(11)$ & $\mathrm{S} 1 \mathrm{~A}-\mathrm{C} 4 \mathrm{~A}-\mathrm{C} 5 \mathrm{~A}$ & $106.53(14)$ \\
\hline $\mathrm{O} 3 \mathrm{~A}-\mathrm{S} 2 \mathrm{~A}-\mathrm{O} 4 \mathrm{~A}$ & $109.48(11)$ & $\mathrm{O} 1 \mathrm{~A}-\mathrm{C} 5 \mathrm{~A}-\mathrm{C} 6 \mathrm{~A}$ & $122.4(2)$ \\
\hline $\mathrm{O} 3 \mathrm{~A}-\mathrm{S} 2 \mathrm{~A}-\mathrm{O} 5 \mathrm{~A}$ & $106.04(11)$ & $\mathrm{O} 1 \mathrm{~A}-\mathrm{C} 5 \mathrm{~A}-\mathrm{C} 4 \mathrm{~A}$ & 119.07(19) \\
\hline $\mathrm{O} 2 \mathrm{~A}-\mathrm{C} 9 \mathrm{~A}-\mathrm{C} 8 \mathrm{~A}$ & $125.06(18)$ & $\mathrm{O} 2 \mathrm{~A}-\mathrm{C} 9 \mathrm{~A}-\mathrm{C} 10 \mathrm{~A}$ & 115.36(19) \\
\hline \multicolumn{4}{|c|}{$10 f$} \\
\hline Bond & $\AA$ & Bond & $\AA$ \\
\hline $\mathrm{S} 1-\mathrm{C} 7$ & $1.7515(12)$ & $\mathrm{N} 1-\mathrm{C} 1$ & $1.3752(16)$ \\
\hline $\mathrm{S} 1-\mathrm{C} 8$ & $1.8058(13)$ & $\mathrm{N} 1-\mathrm{C} 7$ & $1.3554(15)$ \\
\hline $\mathrm{S} 2-\mathrm{C} 10$ & $1.7236(13)$ & $\mathrm{N} 2-\mathrm{C} 6$ & $1.3865(15)$ \\
\hline $\mathrm{S} 2-\mathrm{C} 13$ & $1.7082(16)$ & $\mathrm{N} 2-\mathrm{C} 7$ & $1.3224(15)$ \\
\hline $\mathrm{O} 1-\mathrm{C} 9$ & $1.2162(18)$ & & \\
\hline Angle & $\circ$ & Angle & $\circ$ \\
\hline $\mathrm{C} 7-\mathrm{S} 1-\mathrm{C} 8$ & $100.47(6)$ & $\mathrm{N} 1-\mathrm{C} 7-\mathrm{N} 2$ & $113.39(10)$ \\
\hline $\mathrm{C} 10-\mathrm{S} 2-\mathrm{C} 13$ & $91.62(7)$ & $\mathrm{S} 1-\mathrm{C} 7-\mathrm{N} 1$ & $121.61(8)$ \\
\hline $\mathrm{C} 1-\mathrm{N} 1-\mathrm{C} 7$ & $106.66(9)$ & $\mathrm{S} 1-\mathrm{C} 8-\mathrm{C} 9$ & $113.44(9)$ \\
\hline $\mathrm{C} 6-\mathrm{N} 2-\mathrm{C} 7$ & 104.82(9) & $\mathrm{O} 1-\mathrm{C} 9-\mathrm{C} 10$ & $121.54(12)$ \\
\hline $\mathrm{N} 1-\mathrm{C} 1-\mathrm{C} 2$ & $131.98(11)$ & $\mathrm{O} 1-\mathrm{C} 9-\mathrm{C} 8$ & $122.19(11)$ \\
\hline $\mathrm{N} 1-\mathrm{C} 1-\mathrm{C} 6$ & $105.79(10)$ & $\mathrm{S} 2-\mathrm{C} 10-\mathrm{C} 11$ & $111.21(10)$ \\
\hline $\mathrm{N} 2-\mathrm{C} 6-\mathrm{C} 1$ & 109.33(10) & $\mathrm{S} 2-\mathrm{C} 10-\mathrm{C} 9$ & $119.21(10)$ \\
\hline $\mathrm{N} 2-\mathrm{C} 6-\mathrm{C} 5$ & $130.19(10)$ & $\mathrm{S} 2-\mathrm{C} 13-\mathrm{C} 12$ & $112.46(11)$ \\
\hline $\mathrm{S} 1-\mathrm{C} 7-\mathrm{N} 2$ & $124.83(9)$ & & \\
\hline
\end{tabular}

The tested imidazole and benzimidazole derivatives displayed different levels of growth-inhibitory action toward the examined two breast cancer T4-7D and MCF-7 cell lines. As can be seen in Table 3, the tested imidazole/benzimidazole derivatives were more potent against MCF- 7 cell line $\left(\mathrm{IC}_{50}\right.$ range: $4.53 \pm 0.33-72.64 \pm 4.05 \mu \mathrm{M}$ ) than $\mathrm{T} 4-7 \mathrm{D}$ cell line $\left(\mathrm{IC}_{50}\right.$ range: $\left.8.04 \pm 0.32->100 \mu \mathrm{M}\right)$, with an exception for benzimidazole derivative 10d that displayed enhanced activity against T4-7D cell line $\left(\mathrm{IC}_{50}=8.04 \pm 0.32 \mu \mathrm{M}\right)$ than $\mathrm{MCF}-7$ cell line $\left(\mathrm{IC}_{50}=12.90 \pm 1.04 \mu \mathrm{M}\right)$. 


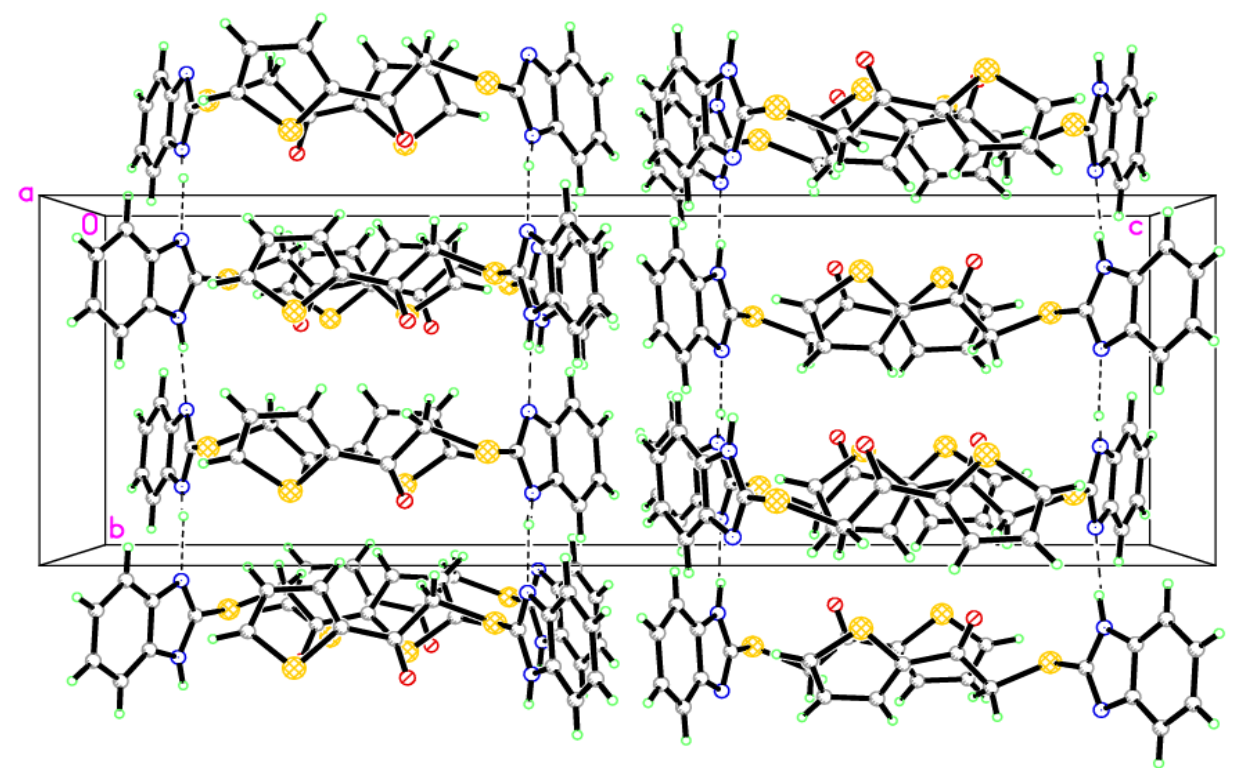

Figure 4. Molecular packing for benzimidazole 10f, showing hydrogen bonds that were drawn as dashed lines along the $b$ axis.

Table 2. Hydrogen-bond geometry $\left(\AA,^{\circ}\right)$ for $9 \mathrm{~b}$ and $10 \mathrm{f} 9 \mathrm{~b}$.

\begin{tabular}{|c|c|c|c|c|}
\hline \multicolumn{5}{|c|}{$9 b$} \\
\hline$D-\mathrm{H} \cdots A$ & $D-\mathrm{H}$ & $H \cdots A$ & $D \cdots A$ & $D-\mathbf{H} \cdots A$ \\
\hline О3А-Н3АА $\cdots$ O5B & 0.8200 & 1.8300 & $2.648(3)$ & 180.00 \\
\hline $\mathrm{O} 4 \mathrm{~B}-\mathrm{H} 4 \mathrm{BC} \cdots \mathrm{O} 6 \mathrm{~A}$ & 0.8200 & 1.8200 & $2.635(3)$ & 179.00 \\
\hline $\mathrm{N} 2 \mathrm{~B}-\mathrm{H} 2 \mathrm{NB} \cdots \mathrm{O} 6 \mathrm{~B}^{\mathrm{i}}$ & $0.77(3)$ & $2.04(3)$ & $2.805(3)$ & $172(2)$ \\
\hline $\mathrm{N} 2 \mathrm{~A}-\mathrm{H} 2 \mathrm{NA} \cdots \mathrm{O} 4 \mathrm{~A}$ & $0.80(3)$ & $1.98(3)$ & $2.762(3)$ & $168(2)$ \\
\hline $\mathrm{N} 1 \mathrm{~A}-\mathrm{H} 1 \mathrm{NA} \cdots \mathrm{O} 5 \mathrm{~A}^{\mathrm{i}}$ & $0.81(3)$ & $1.96(3)$ & $2.769(3)$ & $173(3)$ \\
\hline N1B-H1NB $\cdots$ O3B & $0.83(3)$ & 1.94(3) & $2.768(2)$ & $173(2)$ \\
\hline $\mathrm{C} 1 \mathrm{~A}-\mathrm{H} 1 \mathrm{AA} \cdots \mathrm{O} 2 \mathrm{~A}^{\mathrm{ii}}$ & 0.9300 & 2.5400 & $3.259(3)$ & 134.00 \\
\hline $\mathrm{C} 1 \mathrm{~B}-\mathrm{H} 1 \mathrm{BA} \cdots \mathrm{O} 2 \mathrm{~B}^{\mathrm{iii}}$ & 0.9300 & 2.5400 & $3.274(3)$ & 136.00 \\
\hline $\mathrm{C} 4 \mathrm{~B}-\mathrm{H} 4 \mathrm{BA} \cdots \mathrm{O} 6 \mathrm{~B}^{\mathrm{iv}}$ & 0.9700 & 2.5000 & $3.423(3)$ & 158.00 \\
\hline \multicolumn{5}{|c|}{ Symmetry codes: (i) $\mathrm{x}+1, \mathrm{y}, \mathrm{z}$; (ii) $\mathrm{x}+1, \mathrm{y}+1, \mathrm{z}$; (iii) $\mathrm{x}-1, \mathrm{y}-1, \mathrm{z}$; (iv) $-\mathrm{x}+1,-\mathrm{y}+1,-\mathrm{z}+2$; (v) $\mathrm{x}-1, \mathrm{y}, \mathrm{z}$} \\
\hline \multicolumn{5}{|c|}{$10 \mathrm{f}$} \\
\hline $\mathrm{D}-\mathrm{H} \cdots \mathrm{A}$ & $\mathrm{D}-\mathrm{H}$ & H...A & D...A & $\mathrm{D}-\mathrm{H} \cdots \mathrm{A}$ \\
\hline $\mathrm{N} 1-\mathrm{H} 1 \mathrm{~N} 1 \cdots \mathrm{N} 2^{\mathrm{i}}$ & $0.906(19)$ & $1.88(2)$ & $2.7858(14)$ & $173.7(16)$ \\
\hline \multicolumn{5}{|c|}{ Symmetry code: (i) $-x+1 / 2, y+1 / 2, z$. } \\
\hline
\end{tabular}

As for the growth-inhibitory action against MCF-7 cell line, benzimidazole derivative 10e was the most efficient analogue in the current study possessing better efficiency $\left(\mathrm{IC}_{50}=4.53 \pm 0.33 \mu \mathrm{M}\right)$ than reference drug staurosporine $\left(\mathrm{IC}_{50}=6.67 \pm 0.28 \mu \mathrm{M}\right)$ (Table 3). In addition, compound $10 \mathrm{~d}$ displayed good activity toward MCF-7 cells $\left(\mathrm{IC}_{50}=12.90 \pm 1.04 \mu \mathrm{M}\right)$ that represent two-fold decreased efficiency than staurosporine $\left(\mathrm{IC}_{50}=6.67 \pm 0.28 \mu \mathrm{M}\right)$. In addition, imidazole derivatives $10 \mathrm{a}$ and $10 \mathrm{~b}$ and benzimidazole derivative $10 \mathrm{f}$ possessed moderate growth-inhibitory action toward MCF-7 cell line with $\mathrm{IC}_{50}$ values equal to $42.61 \pm 3.08,31.25 \pm 2.36$, and $23.07 \pm 2.19 \mu \mathrm{M}$, respectively, whereas, imidazole derivative 10c exhibited weak cytotoxic action toward MCF-7 cells $\left(\mathrm{IC}_{50}=6.67 \pm 0.28 \mu \mathrm{M}\right)$. 
Table 3. In vitro anticancer activity of imidazole derivatives $(\mathbf{1 0 a}-\mathbf{c})$ and benzimidazole derivatives (10d-f) against breast cancer T4-7D and MCF-7 cell lines.

\begin{tabular}{|c|c|c|c|}
\hline $\begin{array}{ll} & \mathrm{N} \\
\mathrm{H} & \mathbf{1}\end{array}$ & $\mathbb{1}_{0}^{A r}$ & 10 & \\
\hline \multirow{2}{*}{ Cpd. } & \multirow{2}{*}{ Ar } & \multicolumn{2}{|c|}{$\mathrm{IC}_{50}(\mu \mathrm{M})^{\mathrm{a}}$} \\
\hline & & T-47D & MCF-7 \\
\hline $10 a$ & $\mathrm{C}_{6} \mathrm{H}_{5}$ & $57.68 \pm 0.25$ & $42.61 \pm 3.08$ \\
\hline $10 \mathrm{~b}$ & $4-\mathrm{CH}_{3} \mathrm{O}-\mathrm{C}_{6} \mathrm{H}_{4}$ & $39.32 \pm 0.08$ & $31.25 \pm 2.36$ \\
\hline $10 \mathrm{c}$ & thien2-yl & $\mathrm{NA}^{\mathrm{b}}$ & $72.64 \pm 4.05$ \\
\hline $10 \mathrm{~d}$ & $\mathrm{C}_{6} \mathrm{H}_{5}$ & $8.04 \pm 0.32$ & $12.90 \pm 1.04$ \\
\hline $10 \mathrm{e}$ & $4-\mathrm{CH}_{3} \mathrm{O}-\mathrm{C}_{6} \mathrm{H}_{4}$ & $11.17 \pm 0.04$ & $4.53 \pm 0.33$ \\
\hline $10 f$ & thien-2-yl & $34.94 \pm 0.37$ & $23.07 \pm 2.19$ \\
\hline Staurosporine & - & $7.19 \pm 0.43$ & $6.67 \pm 0.28$ \\
\hline
\end{tabular}

${ }^{\mathrm{a}} \mathrm{IC}_{50} \mathrm{~s}$ calculated as the mean $\pm \mathrm{SD}$ of three separate experiments. ${ }^{\mathrm{b}} \mathrm{NA}$ : Compound showing $\mathrm{IC}_{50}$ value more than $100 \mu \mathrm{M}$.

With regard to the anticancer activity toward breast cancer T4-7D cell line, benzimidazole derivative 10d emerged as the best counterpart in inhibiting growth of the T4-7D cells with comparable activity $\left(\mathrm{IC}_{50}=8.04 \pm 0.32 \mu \mathrm{M}\right)$ to reference drug staurosporine $\left(\mathrm{IC}_{50}=7.19 \pm 0.43 \mu \mathrm{M}\right)$, Table 3 . Besides, benzimidazole derivative 10e efficiently inhibited growth of the T4-7D cells with $\mathrm{IC}_{50}$ value equal to $11.17 \pm 0.04 \mu \mathrm{M}$. Moreover, T4-7D cell line was moderately affected by imidazole derivatives $10 \mathrm{a}$ and $10 \mathrm{~b}$ and benzimidazole derivative $10 \mathrm{f}\left(\mathrm{IC}_{50}=34.94 \pm 0.37,57.68 \pm 0.25\right.$ and $39.32 \pm 0.08 \mu \mathrm{M}$, respectively), whereas, imidazole derivative 10c failed to inhibit the growth of the examined T4-7D cells up to $100 \mu \mathrm{M}$.

Certain structure activity relationships (SARs) should be highlighted from the obtained results (Table 3). Utilization of the benzimidazole scaffold was suggested to be more beneficial for the anticancer activity as evidenced by the enhanced anti-proliferative action of benzimidazole derivatives $10 \mathrm{~d}-\mathrm{f}$ towards both T4-7D $\left(\mathrm{IC}_{50}=8.04 \pm 0.32,11.17 \pm 0.04\right.$ and $34.94 \pm 0.37 \mu \mathrm{M}$, respectively) and MCF-7 cell lines ( $\mathrm{IC}_{50}=12.90 \pm 1.04,4.53 \pm 0.33$ and $23.07 \pm 2.19 \mu \mathrm{M}$, respectively) than their corresponding imidazole counterparts 10a-c (T4-7D; $\mathrm{IC}_{50}=57.68 \pm 0.25,39.32 \pm 0.08$ and $>100 \mu \mathrm{M}$, respectively) (MCF-7; $\mathrm{IC}_{50}=42.61 \pm 3.08,31.25 \pm 2.36$ and $72.64 \pm 4.05 \mu \mathrm{M}$, respectively). Another aspect worth mentioning is that bioisosteric replacement for the pendant phenyl ring with the heterocyclic thiophene, within the imidazole and benzimidazole derivatives, led to dramatic decrease or even abolishment of activity towards the two examined breast cancer T4-7D and MCF-7cell lines. Moreover, para-substitution of the phenyl ring in compounds $10 \mathrm{a}$ and $10 \mathrm{~d}\left(\mathrm{IC}_{50}=42.61 \pm 3.08\right.$ and $12.90 \pm 1.04 \mu \mathrm{M}$, respectively) with the electron-donating methoxy group resulted in compounds $10 \mathrm{~b}$ and $10 \mathrm{e}$ with enhanced anticancer activity against MCF-7cell line $\left(\mathrm{IC}_{50}=31.25 \pm 2.36\right.$ and $4.53 \pm 0.33 \mu \mathrm{M}$, respectively).

\subsection{CDK Inhibitory Action}

\subsubsection{CDK Molecular Modeling}

On account of their promising anticancer actions toward the two tested human breast cancer T4-7D and MCF-7cell lines, benzimidazole derivatives 10d-f were selected to be further investigated for their potential mechanism of action.

First, molecular modeling study was conducted to explore the potential binding interactions and energy scores within CDK2, CDK4/6, CDK7, and CDK9 kinases' active sites. 
The crystal structures of CDK2, CDK4/6, CDK7, and CDK9 were obtained from the Protein Data Bank, PDB IDs: 5EIV [25], 512i [26], 1ua2 [27], and 3lq5 [28], respectively).

In drug design, docking is an inventible technique that enables the prediction of binding between ligand and receptor and is very useful in interpretation of the biological data. However, docking is an error-prone technique and must be validated. Thus, the four co-crystalized ligands in the enzymes were re-docked to their corresponding enzymes and the Root-mean-square deviation (RMSD) values between the docked and co-crystalized ligands were less than 0.6 in both the NW-docking (docking without water) and W-docking (docking with water) (Table S1). The obtained results indicated a reliable docking protocol and guided us to remove the water molecules [29]. In particular, compounds $10 \mathrm{~d}-\mathrm{f}$ achieved a good, acceptable binding affinity only against CDK2 with compound 10e achieving the highest score, $-8.9 \mathrm{Kcal} / \mathrm{mole}$, while compounds $10 \mathrm{~d}$ and $10 \mathrm{f}$ achieved -8.7 and $-8.0 \mathrm{Kcal} / \mathrm{mole}$, respectively (Table 4). Accordingly, the $\mathrm{IC}_{50}$ values will be determined against $\mathrm{CDK} 2$.

Table 4. Docking energy scores in $\mathrm{kcal} / \mathrm{mol}(S)$ for benzimidazoles $\mathbf{1 0 d}-\mathbf{f}$.

\begin{tabular}{ccccc}
\hline \multirow{2}{*}{ Cpd. } & \multicolumn{4}{c}{ Energy Score (S) kcal/mol } \\
\cline { 2 - 5 } & CDK2 & CDK4/6 & CDK7 & CDk9 \\
\hline 10d & -8.7 & -7.2 & -6.6 & -7.3 \\
10e & -8.9 & -7.2 & -6.7 & -7.5 \\
10f & -8.0 & -6.7 & -6.5 & -7.0 \\
\hline
\end{tabular}

Generally, CDK2 has a hydrophobic active site consisting of nonpolar amino acids like valine, leucine, isoleucine, alanine, and phenylalanine. This hydrophobic nature of the active site enabled compounds $10 \mathrm{~d}-\mathrm{f}$ to bind strongly with the enzyme (Figures $5-7$ ). Also, this explains why compounds $10 \mathrm{e}$ and $10 \mathrm{~d}$ had better activity against the enzyme, as they have a more lipophilic phenyl ring than theinyl in compound 10f. Interestingly, compound 10e achieved the highest activity and score, probably due to the methoxy group that forms an additional interaction with the histidine 84 residue. Another aspect worth mentioning is the intramolecular $\mathrm{H}$-bonding between the carbonyl oxygen and the $\mathrm{NH}$ functionality in the benzimidazole ring (Figure 8). This bond is thought to be responsible for the stabilization of the active conformation of benzimidazole derivatives $10 \mathrm{~d}-\mathrm{f}$ within the CDK2 kinase active site. All bonding interactions of benzimidazoles $10 \mathrm{~d}-\mathrm{f}$ within CDK2 active site are summarized in Table 5.

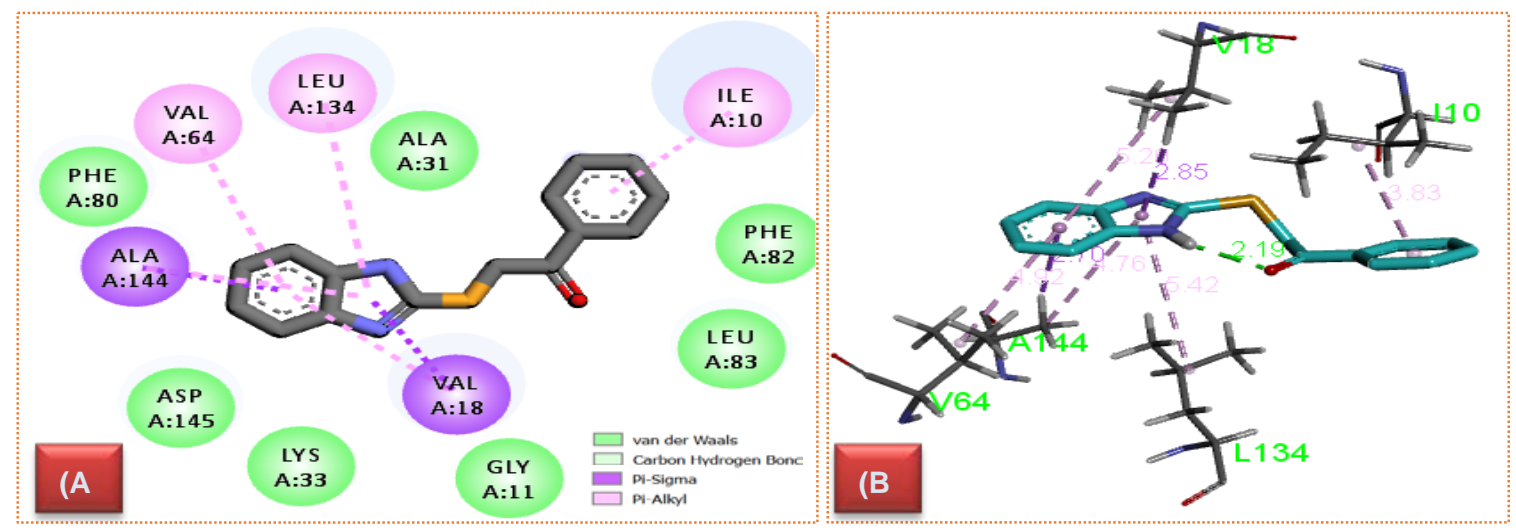

Figure 5. (A) 2D and (B) 3D diagram of benzimidazole 10d displaying its interaction within CDK2 kinase active site (distances in $\AA$ ). 

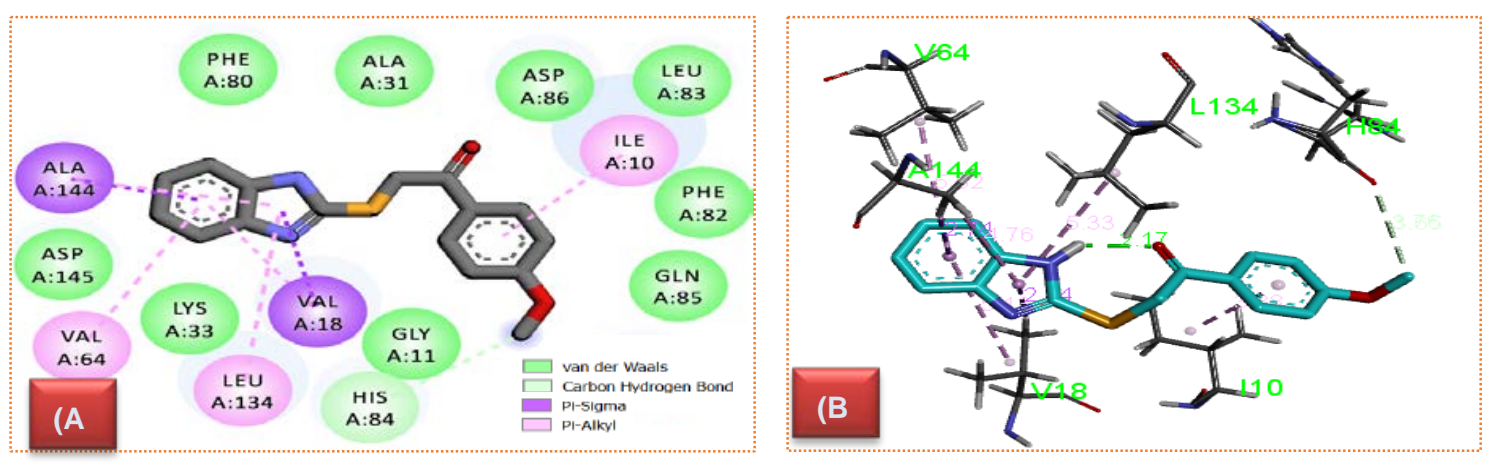

Figure 6. (A) $2 \mathrm{D}$ and (B) 3D diagram of benzimidazole 10e displaying its interaction within CDK2 kinase active site (distances in $\AA$ ).

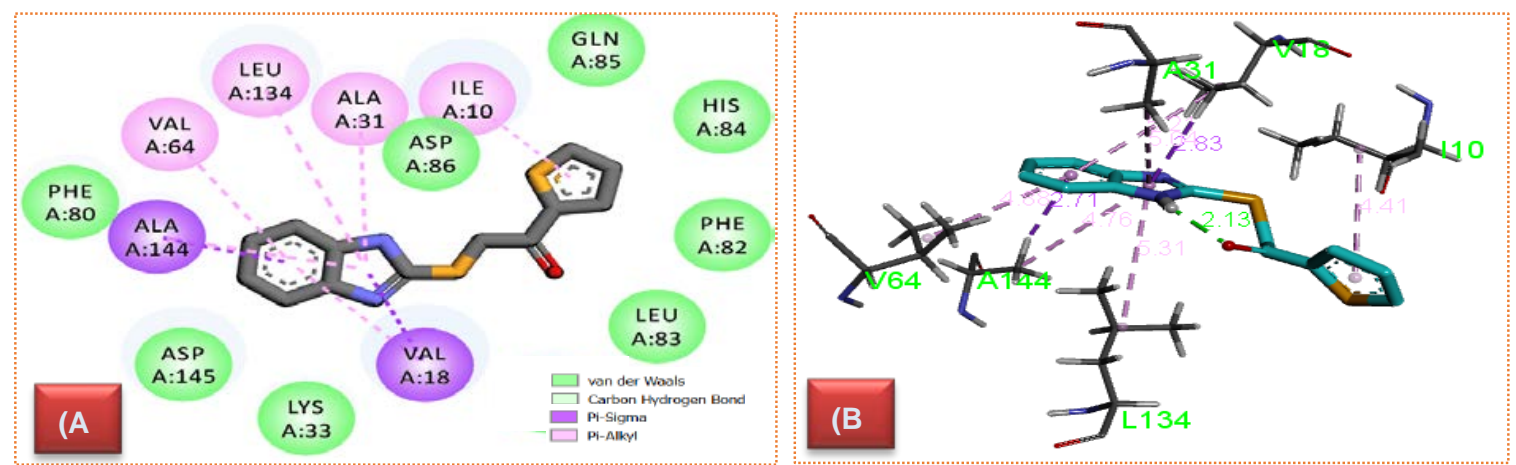

Figure 7. (A) 2D and (B) 3D diagram of benzimidazole 10f displaying its interaction within CDK2 kinase active site (distances in $\AA$ ).

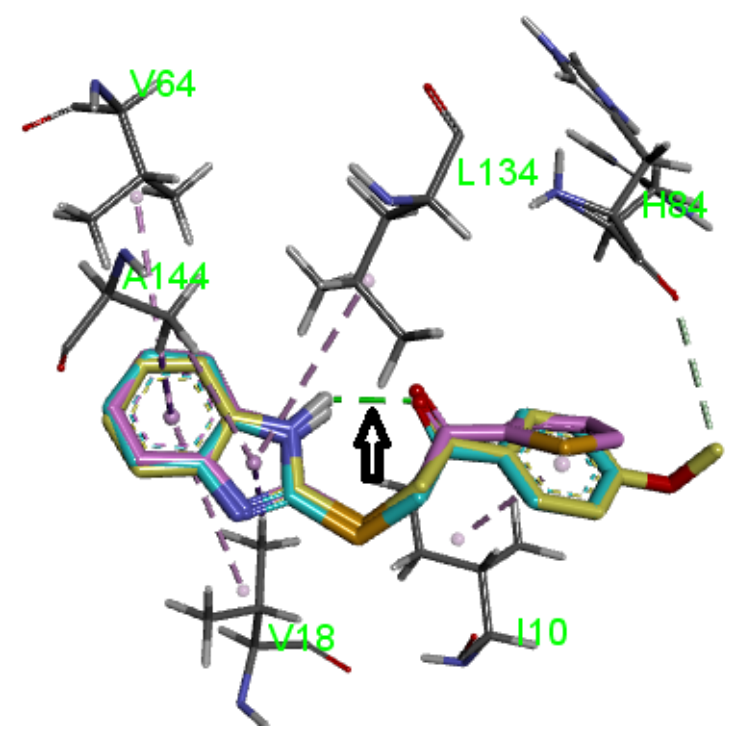

Figure 8. The alignment of the docking results, showing very similar orientation of compounds 10d-f, which are stabilized by an intramolecular hydrogen bond, pointed by black arrow. The 10d is in cyan, 10e is in yellow, and 10f is in pink. 
Table 5. The interaction energy and bonding between benzimidazoles $\mathbf{1 0 d}-\mathbf{f}$ and the CDK2 active site.

\begin{tabular}{cccc}
\hline Cpd. & Bond Type & Involved Amino Acids & Distance \\
\hline \multirow{4}{*}{ 10d } & Pi-Alkyl & Ile10 & 3.83 \\
& Pi-Sigma & Ala144 & 2.70 \\
& Pi-Alkyl & Ala144 & 4.76 \\
& Pi-Alkyl & Leu134 & 5.42 \\
& Pi-Alkyl & Val64 & 4.92 \\
& Pi-Alkyl & Val18 & 5.20 \\
Pi-Sigma & Val18 & 2.85 \\
\hline \multirow{4}{*}{ 10e } & Pi-Alkyl & Ile10 & 3.88 \\
& Pi-Alkyl & Ala144 & 4.76 \\
& Pi-Sigma & Ala144 & 2.47 \\
& Pi-Alkyl & Leu134 & 5.33 \\
& None Classical hydrogen bond & His84 & 3.66 \\
Pi-Alkyl & Val64 & 5.02 \\
Pi-Alkyl & Val18 & 5.13 \\
& Pi-Sigma & Val18 & 2.84 \\
\hline Pi-Alkyl & Ile10 & 4.41 \\
10f & Pi-Alkyl & Val64 & 4.88 \\
& Pi-Alkyl & Ala144 & 4.76 \\
& Pi-Sigma & Ala144 & 2.71 \\
& Pi-Alkyl & Leu134 & 5.31 \\
& Pi-Alkyl & Ala31 & 5.04 \\
& Pi-Alkyl & Val18 & 5.21 \\
& Pi-Sigma & Val18 & 2.88 \\
\hline
\end{tabular}

\subsubsection{In Vitro CDK2 Assay}

In this study, benzimidazole derivatives $10 \mathrm{~d}-\mathrm{f}$ were investigated for their in vitro inhibitory actions against CDK2. The obtained results are presented, as a value $\left(\mathrm{IC}_{50}\right), 50 \%$ inhibition concentration, in Table 6 . The tested benzimidazole derivatives $10 \mathrm{~d}-\mathrm{f}$ exerted moderate to weak inhibitory action against $\mathrm{CDK} 2$, possessing $\mathrm{IC}_{50}$ values equal to $0.89,0.62$, and $1.15 \mu \mathrm{M}$, respectively (Table 6 ). Notably compound 10e, bearing para-methoxy phenyl moiety, was found to be the best CDK2 inhibitor in this study $\left(\mathrm{IC}_{50}=0.62 \mu \mathrm{M}\right)$, whereas bioisosteric replacement for the phenyl ring with the heteroaryl thiophene ring resulted in compound $10 \mathrm{f}$ with the least CDK2 inhibitory action $\left(\mathrm{IC}_{50}=1.15 \mu \mathrm{M}\right)$.

Table 6. $\mathrm{IC}_{50} \mathrm{~s}$ for the inhibitory action for benzimidazole derivatives $\mathbf{1 0 d}-\mathbf{f}$ against CDK2.<smiles>O=C(Br)CSc1nc2ccccc2[nH]1</smiles>

10d-f

\begin{tabular}{ccc}
\hline & & IC $_{50}(\mu \mathrm{M})$ \\
\cline { 3 - 3 } Cpd. & Ar & CDK2 \\
\hline 10d & $\mathrm{C}_{6} \mathrm{H}_{5}$ & 0.89 \\
$\mathbf{1 0 e}$ & $4-\mathrm{CH}_{3} \mathrm{O}-\mathrm{C}_{6} \mathrm{H}_{4}$ & 0.62 \\
$\mathbf{1 0 f}$ & thien-2-yl & 1.15 \\
\hline
\end{tabular}

\subsection{Pharmacokinetics and Druglikeness Properties' Prediction for Benzimidazoles $10 \mathrm{~d}-f$}

Promising candidates should have good pharmacokinetic and pharmacodynamic profiles. Thus, besides determination of the biological activity for benzimidazole derivatives 10d-f, 
their pharmacokinetics, druglikeness, and physicochemical properties were calculated by the SwissADME online tool supplied by the Swiss Institute of Bioinformatics (SIB) [30].

Generally, benzimidazole derivatives 10d-f are predicted to have high GIT absorption. They all are in the human intestinal absorption area in the boiled egg chart [31] (Figure 9). Compound 10e and $10 \mathrm{f}$ have no penetration from the Blood Brain Barrier (BBB) and, thus, no central nervous system (CNS) concerns, unlike compound 10d, which has the probability to penetrate the BBB. Also, Figure 9 revealed that they are not a substrate for PGP (P-glyco protein) and so they fit as anticancer agent. In addition, they are not susceptible for efflux by the P-glyco protein transporters found in many types of cancer as a defense mechanism against anticancer drugs.

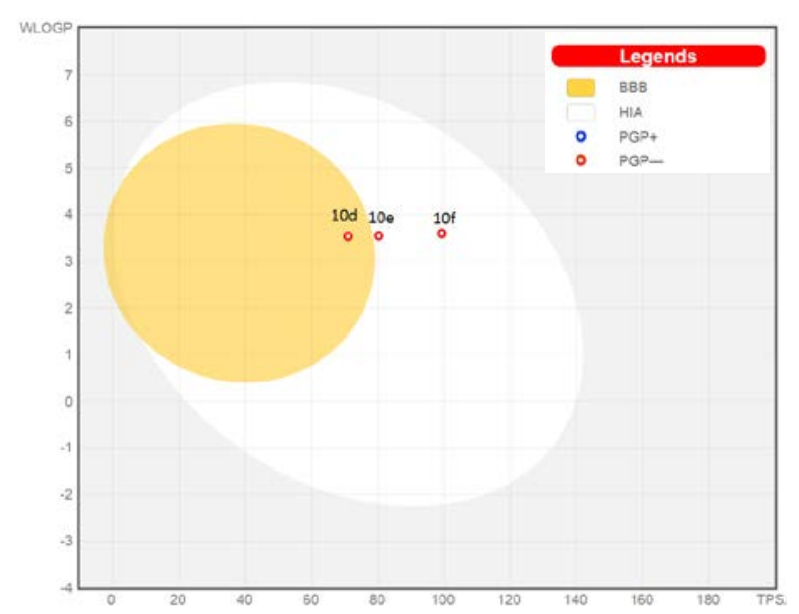

Figure 9. Boiled egg chart, generated from SwissADME online web tool, showing the high oral absorption of benzimidazole derivatives, $\mathbf{1 0 d}-\mathbf{f}$, and the probability of compound $10 \mathrm{~d}$ only to penetrate the BBB.

The bioavailability radar chart explains the high GIT absorption of the three examined compounds 10d-f (Figure 10). The chart contains six critical parameters for oral absorption: FLEX (flexibility), LIPO (lipophilicity), INSATU (saturation), INSOLU (solubility), SIZE, and POLAR (polarity). The chart includes a pink area, representing the optimal range of the six properties, and red lines, representing the predicted physicochemical properties for the explored compounds (Figure 10).

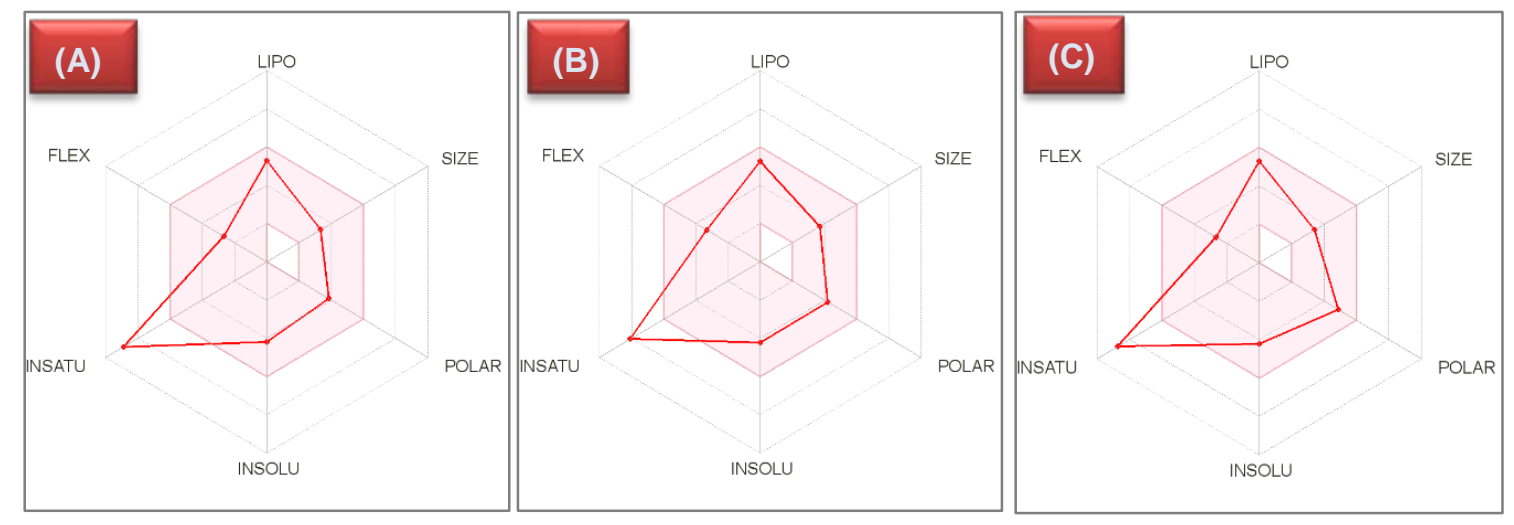

Figure 10. Bioavailability radar chart generated by the aid of SwissADME online web tool for benzimidazole derivatives 10d (A), 10e (B), and 10f (C). Pink area is the range for the optimal property values for oral bioavailability, whereas the red line represents benzimidazoles $10 \mathrm{~d}(\mathrm{~A}), \mathbf{1 0 e}(\mathrm{B})$, and $10 \mathrm{f}$ (C) predicted properties. 
The physicochemical properties of the three benzimidazole derivatives $10 \mathrm{~d}-\mathrm{f}$ were located in the desired range of all the parameters except for the saturation. Penetration through the BBB depends on both the LOGP $\mathrm{o}_{\mathrm{w}}$ and the TPSA (topological polar surface area). The three compounds had LOGP $\mathrm{o}_{\mathrm{w}}$ between 3.71 and 3.76 but only compound $10 \mathrm{~d}$ had the suitable TPSA $\left(71.05 \AA^{2}\right)$ to penetrate the BBB while compounds 10e and 10f had high TPSA values of $80.28 \AA^{2}$ and $99.29 \AA^{2}$, respectively (not suitable for BBB penetration) [32].

The low TPSA value of benzimidazole $10 \mathrm{~d}$ was due to the nonpolar unsubstituted phenyl ring that was more lipophilic than the theinyl and the methoxy substituted phenyl rings found in compounds $10 \mathrm{f}$ and 10e. Compounds 10d-f, as most drugs, were predicted to be metabolized with the liver CYT450 and should be administered alone to minimize the possible drug-drug interactions.

Interestingly, these compounds were found to have no violations in all druglikeness (Veber, Lipinski, Ghose, Muegge, and Egan) rules, and when it comes to medicinal chemistry, all the compounds had neither PAINS (pan assay interference structures) alerts nor structural alerts (Brenks) [33,34]. Table 7 summarizes all the predicted ADME and medicinal chemistry properties for the explored benzimidazole derivatives $10 \mathrm{~d}-\mathrm{f}$. In conclusion, the three compounds, $10 \mathrm{~d}-\mathrm{f}$, demonstrated not only good biological activity but also an acceptable ADME and physicochemical properties, especially benzimidazole 10e.

Table 7. In silico-predicted ADME and druglikeness properties of benzimidazole derivatives 10d-f.

\begin{tabular}{ccccccccccccc}
\hline Cpd. & BвB & GIA & P-gPsubstrate & $\begin{array}{c}\text { CYP2C9 } \\
\text { Inhibitor }\end{array}$ & $\begin{array}{c}\text { CYP2C19 } \\
\text { Inhibitor }\end{array}$ & $\begin{array}{c}\text { CYP1A2 } \\
\text { Inhibitor }\end{array}$ & $\begin{array}{c}\text { CYP2D6 } \\
\text { Inhibitor }\end{array}$ & $\begin{array}{c}\text { CYP3A4 } \\
\text { Inhibitor }\end{array}$ & $\begin{array}{c}\text { PAINS } \\
\text { Alerts }\end{array}$ & $\begin{array}{c}\text { Veber } \\
\text { Violations }\end{array}$ & $\begin{array}{c}\text { Lipinski } \\
\text { Violations }\end{array}$ \\
\hline 10d & Yes & High & No & Yes & Yes & Yes & Yes & Yes & NO & 0 & 0 \\
\hline 10e & No & High & No & Yes & Yes & Yes & Yes & Yes & NO & 0 & 0 \\
\hline $\mathbf{1 0 f}$ & No & High & No & Yes & Yes & Yes & No & Yes & NO & 0 & 0 \\
\hline
\end{tabular}

\section{Materials and Methods}

\subsection{Chemistry}

\subsubsection{General}

Gallenkamp melting point apparatus was utilized to measure melting points for the herein-prepared imidazole/benzimidazoles derivatives, whereas, the Perkin Elmer FT-IR Spectrum BX apparatus was utilized to record the infrared (IR) spectra, as KBr disks. NMR spectra were recorded on a Bruker NMR spectrometer. The ${ }^{1} \mathrm{H} /{ }^{13} \mathrm{C}$ spectra were carried out at $400 / 100 \mathrm{MHz}$, respectively, in deuterated dimethylsulfoxide (DMSO- $d_{6}$ ).

\subsubsection{General Procedures for Synthesis of Salts $9 a-f$}

To a solution of 2-mercaptoimidazole (1a) or 2-mercaptobenzimidazole (1b) (5 mmol) and the appropriate $\beta$-keto ester $5 \mathrm{a}-\mathrm{c}(5 \mathrm{mmol})$ in glacial acetic acid $(10 \mathrm{~mL})$, concentrated sulfuric acid $(50 \mathrm{mmol})$ was added. This reaction mixture was then heated under reflux for one hour, and then allowed to cool to room temperature. The formed precipitate was filtered off, washed with chloroform $(2 \times 5 \mathrm{~mL})$ and then with cold water $(2 \times 3 \mathrm{~mL})$, and dried to yield the corresponding sulfate salts $9 \mathrm{a}-\mathrm{f}$, respectively, which were used in the next step without further purification. The spectroscopic data for benzimidazole sulfates $9 \mathrm{~d}-\mathrm{f}$ were in agreement with that previously reported [20].

2-((2-Oxo2-Phenylethyl)thio)1-H-Imidazol3-ium Hydrogen Sulfate (9a)

Pale yellow powder (yield 89\%); m.p. $246-248{ }^{\circ} \mathrm{C}$; IR: 3420-3172 (2NH + OH) and 1678 (CO); ${ }^{1} \mathrm{H}$ NMR $\delta$ ppm: 4.69 (s, 2H, CH $), 7.01-7.05$ (m, 2H, H-4 and $\mathrm{H}-5$ of imidazole), 7.50-8.03 (m, 5H, Ar-Hs), 8.34, 10.30, $12.72(3 \mathrm{~s}, 3 \mathrm{H}, 2 \mathrm{NH}+\mathrm{OH})$. 
2-((2-(4-Methoxyphenyl)2-Oxoethyl)thio)1-H-Imidazol3-ium Hydrogen Sulfate (9b)

Pale yellow crystals (yield 83\%); m.p. $260-262{ }^{\circ} \mathrm{C}$; IR: 3395-3164 (2NH + OH) and 1684 (CO); ${ }^{1} \mathrm{H}$ NMR $\delta$ ppm: $3.87\left(\mathrm{~s}, 3 \mathrm{H},-\mathrm{OCH}_{3}\right), 4.62\left(\mathrm{~s}, 2 \mathrm{H}, \mathrm{CH}_{2}\right), 7.01-7.07(\mathrm{~m}, 4 \mathrm{H}, \mathrm{H}-4$ and $\mathrm{H}-5$ of imidazole, and $\mathrm{H}-3$ and $\mathrm{H}-5$ of $\left.4-\mathrm{CH}_{3} \mathrm{O}-\mathrm{C}_{6} \mathrm{H}_{4}\right), 8.0\left(\mathrm{~d}, 2 \mathrm{H}, \mathrm{H}-2\right.$ and $\mathrm{H}-6$ of $\left.4-\mathrm{CH}_{3} \mathrm{O}-\mathrm{C}_{6} \mathrm{H}_{4}, J=8.8 \mathrm{~Hz}\right), 8.22,10.0$, $12.75(3 \mathrm{~s}, 3 \mathrm{H}, 2 \mathrm{NH}+\mathrm{OH})$.

2-((2-Oxo2-(Thiophen2-yl)ethyl)thio)1-H-Imidazol3-Ium Hydrogen Sulfate (9c)

White crystals (yield 85\%); m.p. 228-230 ${ }^{\circ} \mathrm{C}$; IR: 3411-3160 (2NH + OH) and $1672(\mathrm{CO}) ;{ }^{1} \mathrm{H}$ NMR Sppm: $4.57\left(\mathrm{~s}, 2 \mathrm{H}, \mathrm{CH}_{2}\right), 7.01-7.04(\mathrm{~m}, 2 \mathrm{H}, \mathrm{H}-4$ and $\mathrm{H}-5$ of imidazole), $7.22(\mathrm{t}, 1 \mathrm{H}, \mathrm{H}-4$ of thiophene, $J=4.4 \mathrm{~Hz}), 7.97(\mathrm{~d}, 1 \mathrm{H}, \mathrm{H}-3$ of thiophene, $J=4.0 \mathrm{~Hz}), 8.02(\mathrm{~d}, 1 \mathrm{H}, \mathrm{H}-5$ of thiophene, $J=4.0 \mathrm{~Hz}), 8.62$, 9.43, $12.0(3 \mathrm{~s}, 3 \mathrm{H}, 2 \mathrm{NH}+\mathrm{OH})$.

\subsubsection{Procedures for Preparation of Imidazole/Benzimidazol-Based Derivatives 10a-f}

To an aqueous solution of sodium hydrogen carbonate, a suspension of the appropriate sulfate salts $9 \mathrm{a}-\mathrm{f}(1 \mathrm{mmol})$ in water $(5 \mathrm{~mL})$ was added. The resulting mixture was allowed to stir at r.t. for three hours. The obtained precipitate was filtered off, washed with cold water $(3 \times 5 \mathrm{~mL})$, dried, and recrystallized from isopropyl alcohol to produce imidazole and benzimidazol derivatives 10a-f, respectively. The spectroscopic data of benzimidazol derivatives $10 \mathrm{~d}-\mathrm{f}$ were in agreement with that previously reported [11].

2-((1H-Imidazol2-yl)thio)1-Phenylethan1-One (10a)

White crystals (yield 70\%); m.p. $173-175{ }^{\circ} \mathrm{C}$; IR: 3375 (NH) and 1673 (CO); ${ }^{1} \mathrm{H}$ NMR $\delta$ ppm: 4.69 (s, $\left.2 \mathrm{H}, \mathrm{CH}_{2}\right), 7.03-7.05\left(\mathrm{~m}, 2 \mathrm{H}, \mathrm{H}-4\right.$ and $\mathrm{H}-5$ of imidazole), 7.54-7.66 (m, 3H, H-3, $\mathrm{H}-4$ and $\mathrm{H}-5$ of $\left.-\mathrm{C}_{6} \mathrm{H}_{5}\right)$, $7.98\left(\mathrm{~d}, 2 \mathrm{H}, \mathrm{H}-2\right.$ and $\mathrm{H}-6$ of $\left.-\mathrm{C}_{6} \mathrm{H}_{5}, J=6.4 \mathrm{~Hz}\right) ;{ }^{13} \mathrm{C}$ NMR $\delta$ ppm: $40.91\left(\mathrm{CH}_{2}\right), 124.32,128.88(2 \mathrm{C})$, $129.21(3 \mathrm{C}), 133.97,135.82,138.42,194.56(\mathrm{C}=\mathrm{O})$.

2-((1H-Imidazol2-yl)thio)1-(4-Methoxyphenyl)ethan1-One (10b)

White crystals (yield 74\%); m.p. $182-184{ }^{\circ} \mathrm{C}$; IR: $3382(\mathrm{NH})$ and $1680(\mathrm{CO}) ;{ }^{1} \mathrm{H}$ NMR $\delta$ ppm: $3.86\left(\mathrm{~s}, 2 \mathrm{H}, \mathrm{CH}_{2}\right), 4.62\left(\mathrm{~s}, 2 \mathrm{H}, \mathrm{CH}_{2}\right), 7.03-7.05(\mathrm{~m}, 4 \mathrm{H}, \mathrm{H}-4$ and $\mathrm{H}-5$ of imidazole, and $\mathrm{H}-3$ and $\mathrm{H}-5$ of $\left.4-\mathrm{OCH}_{3}-\mathrm{C}_{6} \mathrm{H}_{4}\right), 7.98\left(\mathrm{~d}, 2 \mathrm{H}, \mathrm{H}-2\right.$ and $\mathrm{H}-6$ of $\left.4-\mathrm{OCH}_{3}-\mathrm{C}_{6} \mathrm{H}_{4}, J=8.8 \mathrm{~Hz}\right), 12.18(\mathrm{~s}, 1 \mathrm{H}, \mathrm{NH}) ;{ }^{13} \mathrm{C} \mathrm{NMR} \delta$ ppm: $40.73\left(\mathrm{CH}_{2}\right), 56.05\left(\mathrm{OCH}_{3}\right), 114.42(3 \mathrm{C}), 128.65,130.99(3 \mathrm{C}), 138.51,163.89,192.95(\mathrm{C}=\mathrm{O})$.

2-((1H-Imidazol2-yl)thio)1-(Thiophen2-yl)ethan1-One (10c)

White crystals (yield 65\%); m.p. 147-149 ${ }^{\circ} \mathrm{C}$; IR: 3405 (NH) and 1679 (CO); ${ }^{1} \mathrm{H}$ NMR $\delta$ ppm: 4.56 (s, $\left.2 \mathrm{H}, \mathrm{CH}_{2}\right), 7.01-7.04(\mathrm{~m}, 2 \mathrm{H}, \mathrm{H}-4$ and $\mathrm{H}-5$ of imidazole), $7.23(\mathrm{t}, 1 \mathrm{H}, \mathrm{H}-4$ of thiophene, $J=4.4 \mathrm{~Hz}), 7.97$ $(\mathrm{d}, 1 \mathrm{H}, \mathrm{H}-3$ of thiophene, $J=4.0 \mathrm{~Hz}), 8.03(\mathrm{~d}, 1 \mathrm{H}, \mathrm{H}-5$ of thiophene, $J=4.4 \mathrm{~Hz}), 12.32(\mathrm{~s}, 1 \mathrm{H}, \mathrm{NH})$; ${ }^{13} \mathrm{C}$ NMR $\delta$ ppm: $40.60\left(\mathrm{CH}_{2}\right), 129.65(2 \mathrm{C}), 134.67(2 \mathrm{C}), 135.99,138.23,142.63,187.71(\mathrm{C}=\mathrm{O})$.

\subsection{X-ray Crystallographic Analysis}

Both imidazole sulfate-salt $\mathbf{9 b}$ and benzimidazole derivative $10 \mathrm{f}$ were collected as single crystals via slow evaporation from isopropyl alcohol solutions of each pure derivative at ambient temperature. Data were collected by the use of Bruker APEX-II D8 Venture X-ray diffractometer, which was supported with graphite monochromatic $\mathrm{Cu} K \alpha$ radiation, $\lambda=1.54178 \AA$ at 296 (2) $\mathrm{K}$, and Mo $K \alpha$ radiation, $\lambda=0.71073 \AA$ at 293 (2) K, for imidazole sulfate-salt $9 \mathrm{~b}$ and benzimidazole derivative 10f, respectively (Table 8). While the molecular structure for imidazole sulfate-salt $9 \mathrm{~b}$ was crystallized in the Triclinic, $P 1-, a=9.6682(2) \AA, b=11.9413$ (3) $\AA, c=13.1649$ (3) $\AA, \alpha=93.882(2)^{\circ}, \beta=91.557382(1)^{\circ}, \gamma=100 .(2)^{\circ}$, $V=1489.42(6) \AA^{3}, Z=4$, benzimidazole derivative $10 \mathrm{f}$ was crystallized in the orthorhombic, $P b c a$, $a 8.6619$ (2) $\AA, b=9.8244$ (2) $\AA, c=29.7158$ (7) $\AA$, and $V=2528.75$ (10) $\AA^{3}, Z=8$. Cell refinements, as well as data reduction, were performed by the use of Bruker SAINT. SHELXT [35,36] was utilized to 
solve the structures. The final refinements was performed by the full-matrix, least-squares technique with anisotropic thermal data for nonhydrogen atoms on F. CCDC 1524590 and 1429947, comprising the supplementary crystallographic data for imidazole sulfate-salt $9 \mathrm{~b}$ and benzimidazole derivative 10f, were freely available and could be downloaded from the Cambridge Crystallographic Data Centre (http://www.ccdc.cam.ac.uk/data_request/cif).

Table 8. Experimental details for imidazole sulfate-salt $9 \mathbf{b}$ and benzimidazole derivative 10f.

\begin{tabular}{|c|c|c|}
\hline Crystal Data & $9 b$ & $10 \mathrm{f}$ \\
\hline Chemical formula & $\mathrm{C}_{12} \mathrm{H}_{14} \mathrm{~N}_{2} \mathrm{O}_{6} \mathrm{~S}_{2}$ & $\mathrm{C}_{13} \mathrm{H}_{10} \mathrm{~N}_{2} \mathrm{OS}_{2}$ \\
\hline $\mathrm{Mr}$ & 346.37 & 274.35 \\
\hline Temperature (K) & 296 & 293 \\
\hline $\begin{array}{l}\text { Crystal system, } \\
\text { space group }\end{array}$ & Triclinic, P1- & Orthorhombic, $\mathrm{Pbca}$ \\
\hline$a, b, c(\AA)$ & $9.6682(2), 11.9413(3), 13.1649$ (3) & $8.6619(2), 9.8244(2), 29.7158(7)$ \\
\hline V (̊̊) & $1489.42(6)$ & $2528.75(10)$ \\
\hline Z & 4 & 8 \\
\hline$\mu\left(\mathrm{mm}^{-1}\right)$ & 3.54 & 0.41 \\
\hline Radiation type & $\mathrm{Cu} K \alpha$ & Mo $K \alpha$ \\
\hline Crystal size $(\mathrm{mm})$ & $0.60 \times 0.16 \times 0.12$ & $0.48 \times 0.20 \times 0.11$ \\
\hline \multicolumn{3}{|c|}{ Data Collection } \\
\hline Diffractometer & \multicolumn{2}{|c|}{ Bruker APEX-II D8 venture } \\
\hline Absorption correction & \multicolumn{2}{|c|}{ Multi-scan, SADABS Bruker 2014} \\
\hline Tmin, Tmax & \multicolumn{2}{|l|}{$0.226,0.674$} \\
\hline $\mathrm{R}_{\text {int }}$ & \multicolumn{2}{|r|}{0.038} \\
\hline $\begin{array}{l}\text { No. of measured, } \\
\text { independent and observed } \\
{[\mathrm{I}>2 \sigma(\mathrm{I})] \text { reflections }}\end{array}$ & \multicolumn{2}{|r|}{$60453,4406,3778$} \\
\hline \multicolumn{3}{|c|}{ Refinement } \\
\hline $\mathrm{R}\left[F^{2}>2 \sigma\left(F^{2}\right)\right], \mathrm{wR}\left(F^{2}\right), \mathrm{S}$ & $0.038,0.105,1.05$ & $0.039,0.111,0.99$ \\
\hline Reflections Number & 4617 & 4406 \\
\hline Restraints Number & 0 & 0 \\
\hline Parameters Number & 416 & 167 \\
\hline $\mathrm{H}$ atoms treatment & \multicolumn{2}{|c|}{$\mathrm{H}$-atoms were treated by independent and constrained refinement mixture } \\
\hline$\Delta \rho_{\max }, \Delta \rho_{\min }\left(\mathrm{e} \AA^{-3}\right)$ & $0.31-0.32$ & $0.52-0.39$ \\
\hline
\end{tabular}

\subsection{Biological Evaluations}

Experimental procedures adopted for the cytotoxicity [37] and CDK2 [38] assays were performed as reported earlier, and their details are provided in the supporting materials.

\subsection{In Silico Studies}

\subsubsection{Molecular Modeling}

Aiming to identify the potential mechanism of action for the most effective anti-proliferative agents (10d-f), the crystal structures of CDK2, CDK4/6, CDK7, and CDK9 were obtained from the (PDB IDs: 5EIV [25], 512i [26], 1ua2 [27] and 3lq5 [28], respectively). Docking validity was determined by re-docking the co-crystalized into their complexed enzymes, once in presence of water and another 
in water absence. The judgment was based on the calculated RMSD between the docking results and the co-crystalized poses. The docking studies were conducted by Autodock Vina, which demands both receptor and the ligands to be in pdbqt extension thus. M.G.L tools were used to prepare the four enzymes, the co-crystalized and three lead compounds, in the needed format prior to the docking [39]. The obtained docking results were visually inspected by the aid of discovery studio 4.5 visualizer [40]. The selection of the most potential target relied on docking score and interaction within the receptor.

\subsubsection{Pharmacokinetics and Druglikeness Properties' Prediction for Benzimidazoles 10d-f}

Physicochemical and druglikeness properties for benzimidazole derivatives (10d-f) were predicted by SwissADME server, available online [30].

\section{Conclusions}

This study described an unexpected synthetic route for preparation of 2-((imidazol/ benzimidazol2-yl)thio)1-arylethan1-ones 10a-f via reaction of 2-mercaptoimidazole/benzimidazole $2 \mathrm{a}, \mathrm{b}$ with $\beta$-keto esters $6 \mathrm{a}-\mathrm{c}$. The structures for compounds 10a-f were unambiguously confirmed based on NMR spectroscopy and single-crystal X-ray crystallographic analyses. X-ray structure crystal analysis was performed for imidazole sulfate-salt $9 \mathrm{~b}$ and benzimidazole derivative $10 \mathrm{f}$ to confirm their structure, and their data were deposited within the CCDC, with deposition numbers CCDC 1524590 and 1429947, respectively. In vitro anticancer activities for herein-reported imidazole/benzimidazoles $10 \mathrm{a}-\mathrm{f}$ were assessed through a cell-based assay against human breast cancer $\mathrm{T} 4-7 \mathrm{D}$ and MCF-7 cell lines. Benzimidazoles 10d-f exerted better anti-proliferative action towards T4-7D and MCF-7 cell lines than their corresponding imidazole counterparts, 10a-c. Furthermore, CDK2 kinase was suggested as a potential enzymatic target for benzimidazoles $10 \mathrm{~d}-\mathrm{f}$. The obtained results from the in vitro CDK2 assay revealed that compounds $10 \mathrm{~d}-\mathrm{f}$ possess good inhibitory action against CDK2 with $\mathrm{IC}_{50} \mathrm{~s}$ of $0.89,0.62$, and $1.15 \mu \mathrm{M}$, respectively. Besides, the molecular docking study investigated the possible binding pattern and interactions for benzimidazoles $10 \mathrm{~d}-\mathrm{f}$ within CDK2 active site. Finally, several key ADME and druglikeness properties were estimated by the SwissADME online tool. Interestingly, benzimidazoles $10 \mathrm{~d}-\mathrm{f}$ were found to have no violations in all druglikeness (Veber, Lipinski, Ghose, Muegge, and Egan) rules. In addition, they had neither PAINS nor structural alerts (Brenks). In conclusion, benzimidazoles $10 \mathrm{~d}-\mathrm{f}$ demonstrated not only promising anticancer activities but also an acceptable ADME and physicochemical properties, especially benzimidazole 10e.

Supplementary Materials: Supplementary materials are available online at http://www.mdpi.com/2073-4352/10/ 6/446/s1.

Author Contributions: The manuscript was written through contributions of all authors. All authors have given approval to the final version of the manuscript.

Funding: The authors extend their appreciation to the Deanship of Scientific Research at Princess Nourah bint Abdulrahman University for funding this work through the Research Groups Program Grant No. RGP-1440-0025.

Conflicts of Interest: The authors declare no conflict of interest.

\section{References}

1. Yadav, G.; Ganguly, S. Structure activity relationship (SAR) study of benzimidazole scaffold for different biological activities: A mini-review. Eur. J. Med. Chem. 2015, 97, 419-443. [CrossRef] [PubMed]

2. Akhtar, W.; Khan, M.F.; Verma, G.; Shaquiquzzaman, M.; Rizvi, M.A.; Mehdi, S.H.; Akhter, M.; Alam, M.M. Therapeutic evolution of benzimidazole derivatives in the last quinquennial period. Eur. J. Med. Chem. 2016, 126, 705-753. [CrossRef] [PubMed]

3. Tahlan, S.; Kumar, S.; Narasimhan, B. Pharmacological significance of heterocyclic 1 H-benzimidazole scaffolds: A review. BMC Chem. 2019, 13, 101. [CrossRef] [PubMed]

4. Gaba, M.; Mohan, C. Development of drugs based on imidazole and benzimidazole bioactive heterocycles: Recent advances and future directions. Med. Chem. Res. 2016, 25, 173-210. [CrossRef] 
5. Bräse, S. Privileged Scaffolds in Medicinal Chemistry: Design, Synthesis, Evaluation; Royal Society of Chemistry: Cambridge, UK, 2015; Chapter 2; pp. 98-113, ISBN 9781-7-82620-303.

6. Ali, I.; Lone, M.N.; Aboul-Enein, H.Y. Imidazoles as potential anticancer agents. MedChemComm 2017, 8, 1742-1773. [CrossRef]

7. Shrivastava, N.; Naim, M.J.; Alam, M.J.; Nawaz, F.; Ahmed, S.; Alam, O. Benzimidazole scaffold as anticancer agent: Synthetic approaches and structure-activity relationship. Arch. Pharm. 2017, 350, e201700040. [CrossRef]

8. Tahlan, S.; Kumar, S.; Kakkar, S.; Narasimhan, B. Benzimidazole scaffolds as promising antiproliferative agents: A review. BMC Chem. 2019, 13, 66. [CrossRef]

9. Yadav, S.; Narasimhan, B. Perspectives of benzimidazole derivatives as anticancer agents in the new era. Anticancer Agents Med. Chem. 2016, 16, 1403-1425. [CrossRef]

10. Akhtar, M.J.; Yar, M.S.; Sharma, V.K.; Khan, A.A.; Ali, Z.; Haider, M.R.; Pathak, A. Recent Progress of Benzimidazole Hybrids for Anticancer Potential. Curr. Med. Chem. 2019. [CrossRef]

11. Aniket, P.; Shantanu, D.S.; Anagha, O.B.; Ajinkya, P.S. Iodine catalyzed convenient synthesis of 2-aryl1-arylmethyl1- H-benzimidazoles in aqueous media. Int. J. ChemTech Res. 2015, 8, 496-500.

12. Wan, J.P.; Gan, S.F.; Wu, J.M.; Pan, Y. Water mediated chemoselective synthesis of 1,2-disubstituted benzimidazoles using o-phenylenediamine and the extended synthesis of quinoxalines. Green Chem. 2009, 11, 1633-1637. [CrossRef]

13. Di Gioia, M.L.; Cassano, R.; Costanzo, P.; Herrera Cano, N.; Maiuolo, L.; Nardi, M.; Nicoletta, F.P.; Oliverio, M.; Procopio, A. Green synthesis of privileged benzimidazole scaffolds using active deep eutectic solvent. Molecules 2019, 24, 2885. [CrossRef]

14. Herrera Cano, N.; Uranga, J.G.; Nardi, M.; Procopio, A.; Wunderlin, D.A.; Santiago, A.N. Selective and eco-friendly procedures for the synthesis of benzimidazole derivatives. The role of the $\operatorname{Er}(\mathrm{OTf}) 3$ catalyst in the reaction selectivity. Beilstein J. Org. Chem. 2016, 12, 2410-2419. [CrossRef] [PubMed]

15. Sapkal, S.B.; Shelke, K.F.; Sonar, S.S.; Shingate, B.B.; Shingare, M.S. Acidic ionic liquid catalyzed environmentally friendly synthesis of benzimidazole derivatives. Bull. Catal. Soc. India 2009, 2, 78-83.

16. Sharma, J.; Soni, P.K.; Bansal, R.; Halve, A.K. Synthetic Approaches Towards Benzimidazoles by the Reaction of o-Phenylenediamine with Aldehydes Using a Variety of Catalysts: A Review. Curr. Org. Chem. 2018, 22, 2280-2299. [CrossRef]

17. Brain, C.T.; Brunton, S.A. An intramolecular palladium-catalysed aryl amination reaction to produce benzimidazoles. Tetrahedron Lett. 2002, 43, 1893-1895. [CrossRef]

18. Nale, D.B.; Bhanage, B.M. The use of various o-phenylenediamines and N-substituted formamides as C 1 sources in a zinc-catalyzed cyclization in the presence of poly (methylhydrosiloxane) provides benzimidazoles in good yields. Benzoxazole and benzothiazole derivates can also be synthesized. Synlett 2015, 26, 2831-2834.

19. Mahesh, D.; Sadhu, P.; Punniyamurthy, T. Copper (II)-catalyzed oxidative cross-coupling of anilines, primary alkyl amines, and sodium azide using TBHP: A route to 2-substituted benzimidazoles. J. Org. Chem. 2016, 81, 3227-3234. [CrossRef]

20. Abdel-Aziz, H.A.; Ghabbour, H.A.; Eldehna, W.M.; Al-Rashood, S.T.; Al-Rashood, K.A.; Fun, H.K.; Al-Tahhan, M.; Al-Dhfyan, A. 2-((Benzimidazol-2-yl) thio)-1-arylethan-1-ones: Synthesis, crystal study and cancer stem cells CD133 targeting potential. Eur. J. Med. Chem. 2015, 104, 1-10. [CrossRef]

21. Abdel-Aziz, H.A.; Eldehna, W.M.; Ghabbour, H.; Al-Ansary, G.H.; Assaf, A.M.; Al-Dhfyan, A. Synthesis, crystal study, and anti-proliferative activity of some 2-benzimidazolylthioacetophenones towards triple-negative breast cancer MDA-MB4-68 cells as apoptosis-inducing agents. Int. J. Mol. Sci. 2016, 17, 1221. [CrossRef]

22. Al-Ansary, G.H.; Eldehna, W.M.; Ghabbour, H.A.; Al-Rashood, S.T.; Al-Rashood, K.A.; Eladwy, R.A.; Al-Dhfyan, A.; Kabil, M.M.; Abdel-Aziz, H.A. Cancer stem cells CD133 inhibition and cytotoxicity of certain 3-phenylthiazolo [3,2-a]benzimidazoles: Design, direct synthesis, crystal study and in vitro biological evaluation. J. Enzym. Inhib. Med. Chem. 2017, 32, 986-991. [CrossRef] [PubMed]

23. Allen, F.H.; Kennard, O.; Watson, D.G.; Brammer, L.; Orpen, A.G.; Taylor, R. Tables of bond lengths determined by X-ray and neutron diffraction. Part 1. Bond lengths in organic compounds. J. Chem. Soc. Perkins Trans. 2 1987, 12, S1-S19. [CrossRef]

24. Skehan, P.; Storeng, R.; Scudiero, D. New colorimetric cyto-toxicity assay for anticancer-drug screening. J. Natl. Cancer Inst. 1990, 82, 1107-1112. [CrossRef] [PubMed] 
25. Ayaz, P.; Andres, D.; Kwiatkowski, D.A.; Kolbe, C.C.; Lienau, P.; Siemeister, G.; Lucking, U.; Stegmann, C.M. Conformational Adaption May Explain the Slow Dissociation Kinetics of Roniciclib (BAY 1000394), a Type I CDK Inhibitor with Kinetic Selectivity for CDK2 and CDK9. ACS Chem. Biol. 2016, 11, 1710-1719. [CrossRef]

26. Chen, P.; Lee, N.V.; Hu, W.; Xu, M.; Ferre, R.A.; Lam, H.; Bergqvist, S.; Solowiej, J.; Diehl, W.; He, Y.A.; et al. Spectrum and Degree of CDK Drug Interactions Predicts Clinical Performance. Mol. Cancer Ther. 2016, 15, 2273-2281. [CrossRef]

27. Lolli, G.; Lowe, E.D.; Brown, N.R.; Johnson, L.N. The Crystal Structure of Human CDK7 and Its Protein Recognition Properties. Structure 2004, 12, 2067-2079. [CrossRef]

28. Bettayeb, K.; Baunbak, D.; Delehouze, C.; Loaec, N.; Hole, A.J.; Baumli, S.; Endicott, J.A.; Douc-Rasy, S.; Benard, J.; Oumata, N.; et al. CDK Inhibitors Roscovitine and CR8 Trigger Mcl1- Down-Regulation and Apoptotic Cell Death in Neuroblastoma Cells. Genes Cancer 2010, 1, 369-380. [CrossRef]

29. Wei, Y.; Li, J.; Qing, J.; Huang, M.; Wu, M.; Gao, F.; Lin, J. Discovery of novel hepatitis C virus NS5B polymerase inhibitors by combining random forest, multiple e-pharmacophore modeling and docking. PLoS ONE 2016, 11, e0148181. [CrossRef]

30. Daina, A.; Michielin, O.; Zoete, V. SwissADME: A free web tool to evaluate pharmacokinetics, drug-likeness and medicinal chemistry friendliness of small molecules. Sci. Rep. 2017, 7, 42717. [CrossRef]

31. Daina, A.; Zoete, V. A BOILED-Egg to predict gastrointestinal absorption and brain penetration of small molecules. ChemMedChem 2016, 11, 1117-1121. [CrossRef]

32. Ertl, P.; Rohde, B.; Selzer, P. Fast Calculation of Molecular Polar Surface Area as a Sum of Fragment-Based Contributions and Its Application to the Prediction of Drug Transport Properties. J. Med. Chem. 2000, 43, 3714-3717. [CrossRef] [PubMed]

33. Baell, J.B.; Holloway, G.A. New substructure filters for removal of pan assay interference compounds (PAINS) from screening libraries and for their exclusion in bioassays. J. Med. Chem. 2010, 53, 2719-2740. [CrossRef] [PubMed]

34. Veber, D.F.; Johnson, S.R.; Cheng, H.Y.; Smith, B.R.; Ward, K.W.; Kopple, K.D. Molecular properties that influence the oral bioavailability of drug candidates. J. Med. Chem. 2002, 45, 2615-2623. [CrossRef] [PubMed]

35. Sheldrick, G.M. A short history of SHELX. Acta Crystallogr. Sect. A 2008, 64, 112-122. [CrossRef]

36. Sheldrick, G.M. SHELXTL-PC (Version 5.1); Siemens Analytical Instruments, Inc.: Madison, WI, USA, 1997.

37. Sabt, A.; Abdelhafez, O.M.; El-Haggar, R.S.; Madkour, H.M.; Eldehna, W.M.; El-Khrisy, E.E.D.A.; Abdel-Rahman, M.A.; Rashed, L.A. Novel coumarin-6-sulfonamides as apoptotic anti-proliferative agents: Synthesis, in vitro biological evaluation, and QSAR studies. J. Enzym. Inhib. Med. Chem. 2018, 33, 1095-1107. [CrossRef]

38. Said, M.A.; Eldehna, W.M.; Nocentini, A.; Fahim, S.H.; Bonardi, A.; Elgazar, A.A.; Kryštof, V.; Soliman, D.H.; Abdel-Aziz, H.A.; Gratteri, P.; et al. Sulfonamide-based ring-fused analogues for CAN508 as novel carbonic anhydrase inhibitors endowed with antitumor activity: Design, synthesis, and in vitro biological evaluation. Eur. J. Med. Chem. 2020, 189, 112019. [CrossRef]

39. Trott, O.; Olson, A.J. AutoDock Vina: Improving the speed and accuracy of docking with a new scoring function, efficient optimization and multithreading. J. Comp. Chem. 2010, 31, 455-461. [CrossRef]

40. Available online: https://3dsbiovia.com/resource-center/downloads/ (accessed on 6 March 2020).

(C) 2020 by the authors. Licensee MDPI, Basel, Switzerland. This article is an open access article distributed under the terms and conditions of the Creative Commons Attribution (CC BY) license (http://creativecommons.org/licenses/by/4.0/). 